\title{
Error Estimates for the Multidimensional Two-Phase Stefan Problem
}

\author{
By Joseph W. Jerome and Michael E. Rose
}

\begin{abstract}
In this paper we derive rates of convergence for regularizations of the multidimensional two-phase Stefan problem and use the regularized problems to define backward-difference in time and $C^{0}$ piecewise-linear in space Galerkin approximations. We find an $L^{2}$ rate of convergence of order $\sqrt{\varepsilon}$ in the $\varepsilon$-regularization and an $L^{2}$ rate of convergence of order $\left(h^{2} / \varepsilon+\Delta t / \sqrt{\varepsilon}\right)$ in the Galerkin estimates which leads to the natural choices $\varepsilon \sim h^{4 / 3}$, $\Delta t \sim h^{4 / 3}$, and a resulting $O\left(h^{2 / 3}\right) L^{2}$ rate of convergence of the numerical scheme to the solution of the differential equation. An essentially $O(h)$ rate is demonstrated when $\varepsilon=0$ and $\Delta t \sim h^{2}$ in our Galerkin scheme under a boundedness hypothesis on the Galerkin approximations. The latter result is consistent with computational experience.
\end{abstract}

1. Introduction. Given a smoothly bounded domain $\Omega \subset \mathbf{R}^{N}$, we consider the equation, in distribution form,

$$
\frac{\partial H(u)}{\partial t}-\Delta u+f(u)=0
$$

on a space-time domain $D=\Omega \times\left(0, T_{0}\right]$ subject to the Neumann boundary condition on $\partial \Omega$

$$
\frac{\partial u}{\partial \nu}=0, \quad \nu=\text { outward normal, }
$$

and the initial condition

$$
\left.H(u)\right|_{t=0}=H\left(u_{0}\right) .
$$

Here $H(\cdot)$ is the discontinuous enthalpy function, $f$ is a Lipschitz continuous real function, and the initial function $u_{0}$ is a bounded continuous function satisfying $u_{0} \in H^{1}(\Omega) \cap W^{2,1}(\Omega)$ with $H\left(u_{0}\right) \in L^{\infty}(\Omega)$. These hypotheses will be maintained throughout the paper, although not all the results to follow require the full strength of the hypotheses. (1.1i) is the transform of the standard equation ([2], [3], [23], [31], [40])

$$
\frac{\partial}{\partial t}(\sigma+s)-\nabla \cdot(k \nabla \theta)+g(\theta)=0, \quad \sigma(\theta)=\int_{0}^{\theta} c(\xi) d \xi,
$$

where we set

$$
u=\int_{0}^{\theta} k(\xi) d \xi=K(\theta)
$$

Received June 9, 1980; revised November 23, 1981 and March 15, 1982.

1980 Mathematics Subject Classification. Primary 65N15, 65N30; Secondary 35K55, 35K65. 
via the Kirchhoff transformation. In (1.2) we assume that $k$ is bounded above zero and that $c, s$, and $k$ are piecewise smooth functions of the temperature $\theta$ discontinuous at the nominal change of phase temperature 0 ; these represent the specific heat, the latent energy content, and thermal conductivity, respectively. Writing

$$
Q(\theta)=\sigma(\theta)+s(\theta),
$$

we define $H=Q \circ K^{-1}$. We assume that $H$ is a monotone increasing function, $C^{1}$ in $(-\infty, 0)$ and $(0, \infty)$ such that $H^{\prime}(0-)$ and $H^{\prime}(0+)$ exist, with a jump discontinuity of height $A$ at 0 and derivative satisfying

$$
0<\lambda \leqslant H^{\prime}(\xi) \leqslant \mu<\infty, \quad \xi \neq 0 .
$$

The normalization is chosen so that

$$
H(0-)=0
$$

and the jump condition takes the form

$$
H(0+)=A>0 .
$$

Equation (1.1i) has been used to model heat transfer in permafrost by $\mathrm{J}$. Wheeler ([39], [40]) and others. It is applicable when the partially frozen soil is saturated, i.e., when liquid water and ice jointly occupy the entire void space of the porous medium. Heat transfer due to convection has been ignored and the underlying porous medium is assumed to be nondeformable, so that frost heave and subsidence are not taken into account.

Unlike the classical Stefan problem, which concerns the freezing of bulk water, the modelling of heat transfer in permafrost must account for the fact that liquid water and ice coexist at a bracket of subzero temperatures. In this case, zero degrees represents the maximum, though not unique, temperature at which both phases coexist in the soil. The constant $A$ represents the product of the heat of fusion $B$ with that fraction of moisture content solidified at the nominal freezing point. The function $s$ describes the latent energy associated with various moisture content percentages as a function of temperature and is here normalized by translation by $A$ so that $s(0-)=0$. Minor modifications permit the inclusion of the multi-phase Stefan problem; what is required is a piecewise smooth function $H$ satisfying a condition like (1.5i) between successive jump discontinuities. For simplicity of analysis only, we have chosen a perfectly insulated boundary condition and have provided a Lipschitz body heating term $g$ in the equation (1.2) which transforms via $f=g \circ K^{-1}$.

Let $D_{1}=\{u<0\}, S=\{u=0\}$, and $D_{2}=\{u>0\}$. Then the system (1.1) is formally equivalent to the pointwise form of Eq. (1.1) on $D_{1} \cup D_{2}$ adjoined to the formally specified conditions

$$
\begin{aligned}
K^{-1}(u)=0 \quad \text { on } S, \\
-\left[k \frac{\partial K^{-1}(u)}{\partial \pi \nu}\right]=A \cos \left(\nu, 1_{t}\right), \quad \nu=\text { outward normal of } D_{2},
\end{aligned}
$$

$\pi \nu=$ the projection of $\nu$ into the plane of $\Omega$, where $\left[k \partial K^{-1}(u) / \partial \pi \nu\right]$ represents the discontinuity of $k \partial K^{-1}(u) / \partial \pi \nu$ across the free boundary interface $S$ directed from 
$D_{2}$ to $D_{1}$; these assert temperature equilibrium on $S$ and conservation of energy across $S$. For simplicity, we have written (1.6) as though $S$ were a "surface"; it is of course possible to generalize (1.6) to the case where $S$ has positive measure. We also note that the case $A=0, B \neq 0$ is physically possible (cf. [2, pp. 94-99]) but less interesting since it leads to standard models (cf. sequel) for which $k \nabla \theta$ is continuous across $S$. Incidentally, if $S$ is the zero set of a smooth function $\phi$, then multiplication of (1.6ii) by $|\nabla \phi|$ gives the familiar relation connecting the velocity of the front and the inner product of $\nabla \phi$ with the jump in the vector flux. The relations (1.1) and (1.6) lead directly to the weak solution formulation given by (cf. [25])

Definition 1.1. Suppose the initial functions $u_{0} \in H^{1}(\Omega), H\left(u_{0}\right) \in L^{2}(\Omega)$ are specified. Then a function $u \in L^{\infty}\left(0, T_{0} ; H^{1}(\Omega)\right)$ with $H(u)$ satisfying

$$
\begin{gathered}
H(u(x)) \in[0, A] \quad \text { if } u(x)=0, \\
H(u) \in L^{2}(D) \cap H^{1}\left(0, T_{0} ;\left(H^{1}(\Omega)\right)^{\prime}\right),
\end{gathered}
$$

is said to be a weak solution of the two-phase Stefan problem (1.1), (1.6) if the relation

$$
\begin{aligned}
\int_{D}[H(u) & \left.\frac{\partial \zeta}{\partial t}-\nabla u \cdot \nabla \zeta-f(u) \zeta\right] d x d \tau \\
& -\int_{\Omega \times\left\{T_{0}\right\}} H(u) \zeta(x, t) d x+\int_{\Omega \times\{0\}} H\left(u_{0}\right) \zeta(x, 0)=0
\end{aligned}
$$

holds for all $\zeta \in \mathcal{C}$ where $D=\Omega \times\left(0, T_{0}\right)$ and

$$
\bigodot=L^{\infty}\left(0, T_{0} ; H^{1}(\Omega)\right) \cap H^{1}\left(0, T_{0} ; L^{2}(\Omega)\right)
$$

Remark 1.1. The existence of weak solutions for such equations can be deduced from the work of Kamenomostskaja [20] and Friedman [15], although neither author considers a term $f(\cdot)$, Neumann boundary conditions, or the existence of the time derivative as an $L^{2}$ function which is necessary for our work. Minor modifications of [17], where a Dirichlet boundary condition is specified, leads to the existence of a solution $u$ satisfying $u_{t} \in L^{2}(D)$, i.e., $u \in \mathcal{C}$. Recent work of Caffarelli and Evans [8] and DiBenedetto [12] has demonstrated that essentially bounded weak solutions are continuous. We remark that our characterization of weak solutions must be modified in our analysis of numerical procedures. This is due to the fact that the estimates to follow require a satisfactory substitute for the pointwise equations. This substitute requires the introduction of a bounded linear operator $T$ from $F=\left(H^{1}(\Omega)\right)^{\prime}$ to $H^{1}(\Omega)$ which is given by an inverse of $-\Delta$ subject to Neumann boundary data. The reader may conveniently think of the equivalent "lifted" relation as an abstract integral equation. The mapping $T$ has been used to obtain error estimates for Galerkin approximations for linear parabolic equations by Bramble, Schatz, Thomée, and Wahlbin [4] and has been used by the second author to analyze finite element methods for degenerate parabolic equations arising in fluid flows in a porous medium [29]. Finally, for essentially bounded weak solutions, the second integral in (1.8i) has the usual meaning. More generally, it has a duality interpretation. 
Definition 1.2. Given $l \in F=\left(H^{1}(\Omega)\right)^{\prime}$, we define the element $w=T l \in H^{1}(\Omega)$ as the uniquely specified element satisfying the weak form of the linear elliptic problem,

$$
\begin{array}{ll}
\text { (i) }-\Delta w=l-\frac{1}{|\Omega|}\langle l, 1\rangle & \text { on } \Omega, \\
\text { (ii) } \frac{\partial w}{\partial \nu}=0 & \text { on } \partial \Omega, . \\
\text { (iii) } \int_{\Omega} w=\langle l, 1\rangle, &
\end{array}
$$

where $\langle\cdot, \cdot\rangle$ represents the duality pairing on $F \times H^{1}(\Omega)$. Here, $-\Delta$ is viewed as a continuous linear mapping from $H^{1}(\Omega)$ onto $F$.

Remark 1.2 . It is easily seen that $T$ is the Riesz map, or, equivalently, that $w=T l$ is the Riesz representer of $l$, when the inner product

$$
(v, w)_{H^{1}(\Omega)}=\int_{\Omega} \nabla v \cdot \nabla w+\frac{1}{|\Omega|} j(v) j(w)
$$

is employed; here $j(v)=\int_{\Omega} v$, and we have

$$
\langle l, v\rangle=\int_{\Omega} \nabla T l \cdot \nabla v+\frac{1}{|\Omega|} j(T l) j(v) .
$$

Note that the norm induced by (1.10) is equivalent to the standard norm on $H^{1}(\Omega)$ (cf. Sobolev [35]). We shall use (1.10) in this paper. The linear mapping $T$, whose restriction to $L^{2}(\Omega)$ is positive-definite and selfadjoint, induces the following norm on $F$ equivalent to that induced by the standard duality norm:

$$
\|l\|_{F}=\{\langle l, T l\rangle\}^{1 / 2} \text {. }
$$

It is not possible, in general, to identify $F$ with a subspace of $H^{-1}(\Omega)$, since $H_{0}^{1}(\Omega)$ is not dense in $H^{1}(\Omega)$.

Proposition 1.1. Let $u$ be a weak solution of the Stefan problem satisfying Definition 1.1 with $u_{t} \in L^{2}(D)$. Then $T H(u) \in H^{2}(D)$, and the equation

$$
\frac{\partial T H(u)}{\partial t}+u+T f(u)=\frac{1}{|\Omega|} \int_{\Omega} u
$$

holds a.e. in $\Omega$ for each $0<t<T_{0}$. Moreover, $\left.T H(u)\right|_{t=0}=T H\left(u_{0}\right)$.

Proof. For $\xi \in C_{0}^{\infty}(D)$, let $\zeta=T \xi$ in (1.8). Using the commutative relation $((\partial / \partial t) T-T \partial / \partial t) \xi=0$ (cf. (1.11)) and the selfadjointness of $T$, we obtain

$$
\int_{D} T H(u) \frac{\partial \xi}{\partial t} d x d \tau=\int_{D}[u+T f(u)] \xi d x d \tau-\frac{1}{|\Omega|} \int_{D} j(u) \xi d x d \tau,
$$

which shows that the distributional derivative $\partial T H(u) / \partial t$ is equal to $-u-T f(u)+$ $j(u) /|\Omega|$. Since $H(u) \in L^{2}(D)$, it follows that $\partial^{2} T H(u) / \partial x_{i}^{2} \in L^{2}(D), i=1, \ldots, N$, and the remaining second order partial derivatives are in $L^{2}(D)$ from the properties of $u$. It follows that $T H(u) \in H^{2}(D)$. In particular, (1.14) can be integrated by parts to obtain (1.13) since $\xi$ is arbitrary and the left-hand side of (1.13) is continuous in $t$ as a mapping into $L^{2}(\Omega)$. The final statement follows similarly. 
Remark 1.3. The first numerical work on the two-phase Stefan problem of which we are aware, in which the enthalpy formulation was a basic starting point, was the constructive existence analysis of [20], based on an explicit differencing scheme, and the paper of Milton Rose [30]. During the 1960's there appeared two papers in the Russian literature modelled on [20], viz., Samarskii and Moiseenko [32] and Budak, Solev'eva and Uspenskii [7]. The advantage of the enthalpy formulation of course is that explicit tracking of the free boundary is unnecessary. Prior to Meyer's work in [26], in which an implicit scheme was analyzed in conjunction with smoothing, there were earlier papers by Solomon [36] and Lazaridis [24]. A subsequent finite element analysis was carried out by Ciavaldini [10]. The present work combines a new regularity analysis for solutions of (1.8), estimation of error in the smoothing, and the rate of convergence of the continuous Galerkin and fully discrete Galerkin schemes.

Finally, we introduce the smoothing of [18]. For $\varepsilon>0$ we define

(i) $w_{\varepsilon}(\xi)=H^{\prime}(\xi), \quad \xi<0, \xi \geqslant \varepsilon$,

(ii) $w_{\varepsilon}(\xi)=q_{\varepsilon}(\xi), \quad 0 \leqslant \xi \leqslant \varepsilon$.

Here $q_{\varepsilon}$ is the uniquely determined quadratic polynomial satisfying

$$
q_{\varepsilon}(0)=H^{\prime}(0-), \quad q_{\varepsilon}(\varepsilon)=H^{\prime}(\varepsilon), \quad \int_{0}^{\varepsilon} q_{\varepsilon}(\xi) d \xi=A .
$$

$H_{\varepsilon}$ is defined by

$$
H_{\varepsilon}(\xi)=\int_{0}^{\xi} w_{\varepsilon}(\zeta) d \zeta
$$

Remark 1.4. It was shown in [18] that, for $0<\varepsilon \leqslant \varepsilon_{0}$, some $\varepsilon_{0} \leqslant 1, H_{\varepsilon}$ is a continuously differentiable Lipschitz function on $\mathbf{R}$ satisfying

$$
0<\lambda \leqslant H_{\varepsilon}^{\prime}(\xi) \leqslant \gamma / \varepsilon, \quad \xi \in \mathbf{R} .
$$

Moreover, $H_{\varepsilon}$ and $H_{\varepsilon}^{\prime}$ converge uniformly to $H$ and $H^{\prime}$, respectively, on compact sets excluding 0 , and $H^{\prime}$ and $H_{\varepsilon}^{\prime}$ agree off the interval $[0, \varepsilon]$. Let $J=H^{-1}$ and $J_{\varepsilon}=H_{\varepsilon}^{-1}$. Since $\left|H(\xi)-H_{\varepsilon}(\xi)\right| \leqslant \mu \varepsilon$ for $|\xi| \geqslant \varepsilon$, we have $\left|J_{\varepsilon}(\eta)-J(\eta)\right| \leqslant$ $(1+\mu / \lambda) \varepsilon$ for $\eta \in \mathbf{R}$. Here $J$ is a Lipschitz continuous function.

Remark 1.5. At various points in this paper we shall use the inequality

$$
\left\|v\left(t_{0}\right)\right\|_{X} \leqslant\|v\|_{L^{\infty}\left(0, T_{0} ; X\right)}, \quad 0 \leqslant t_{0} \leqslant T_{0},
$$

for $v \in L^{\infty}\left(0, T_{0} ; X\right)$. Although such an inequality does not hold in general, we shall apply it only when $v:\left[0, T_{0}\right] \rightarrow X$ is continuous or when the supremum holds over the entire interval $\left[0, T_{0}\right]$. We shall also assume for simplicity that $f(0)=0$.

2. Regularity Theory. We begin by defining a class of regularized problems via the enthalpy smoothing (1.17).

Definition 2.1. Let $H_{\varepsilon}$ be defined by (1.17) for $0<\varepsilon \leqslant \varepsilon_{0}$. Then $u^{\varepsilon}$ is the unique solution of the parabolic boundary value problem

$$
\begin{array}{ll}
\text { (i) } \frac{\partial H_{\varepsilon}\left(u^{\varepsilon}\right)}{\partial t}-\Delta u^{\varepsilon}+f\left(u^{\varepsilon}\right)=0 & \text { on } \Omega \times\left(0, T_{0}\right] \\
\text { (ii) } \frac{\partial u^{\varepsilon}}{\partial \nu}=0 & \text { on } \partial \Omega \times\left(0, T_{0}\right] \\
\text { (iii) } u^{\varepsilon}(x, 0)=u_{0}(x) & \text { on } \Omega .
\end{array}
$$


Remark 2.1. There exists a unique solution $u^{\varepsilon}$ of (2.1) satisfying

$$
u^{\varepsilon} \in \mathscr{Q}=H^{1}\left(0, T_{0} ; L^{2}(\Omega)\right) \cap L^{\infty}\left(0, T_{0} ; H^{1}(\Omega)\right) \cap L^{2}\left(0, T_{0} ; H^{2}(\Omega)\right) .
$$

The method of lines yields the regularity described by the first two spaces in (2.2), where $u^{\varepsilon}$ is a weak solution of (2.1) (cf. (1.8)). Using (1.13), we see that

$$
\frac{\partial T H_{\varepsilon}\left(u^{\varepsilon}\right)}{\partial t}+u^{\varepsilon}+T f\left(u^{\varepsilon}\right)=\frac{1}{|\Omega|} \int_{\Omega} u^{\varepsilon} d x \quad \text { a.e. in } \Omega, 0<t<T_{0},
$$

and the commutation of $T$ and $\partial / \partial t$ reveals that $u^{\varepsilon}$ is in the third class described by (2.2). Note that here we use the fact that $\partial H_{\varepsilon}\left(u^{\varepsilon}\right) / \partial t \in L^{2}(D)$. In particular, since (2.2) holds, $\Delta u^{\varepsilon} \in L^{2}(D)$, and integration by parts in the weak formulation is valid, we conclude that (2.1) holds in the standard way. In fact, we see that (2.1i) holds a.e. in $\Omega$, for all $0<t<T_{0}$, by operating on (2.3) with $-\Delta$. Under the hypothesis that $u_{0} \in L^{\infty}(\Omega)$, it follows that $u^{\varepsilon} \in L^{\infty}(D)$, and in this case it is known that $u^{\varepsilon}$ is Hölder continuous (cf. Ladyženskaya, Solonnikov and Ural'ceva [23, pp. 417-423]).

Proposition 2.1. There exists a constant $C$ such that the relations

(i) $\left\|H_{\varepsilon}\left(u^{\varepsilon}\right)\right\|_{L^{\infty}\left(0, T_{0} ; L^{2}(\Omega)\right)} \leqslant C$,

(ii) $\left\|u_{t}^{\varepsilon}\right\|_{L^{2}(D)}+\left\|\nabla u^{\varepsilon}\right\|_{L^{\infty}\left(0, T_{0} ; L^{2}(\Omega)\right)} \leqslant C$,

(iii) $\left\|\left[H_{\varepsilon}\left(u^{\varepsilon}\right)\right]_{t}\right\|_{L^{2}(D)} \leqslant C / \sqrt{\varepsilon}$,

(iv) $\left\|H_{\varepsilon}\left(u^{\varepsilon}\right)\right\|_{L^{2}\left(0, T_{0} ; H^{1}(\Omega)\right)} \leqslant C / \sqrt{\varepsilon}$,

hold for $0<\varepsilon \leqslant \varepsilon_{0}$. In (2.4i), $C$ depends continuously upon $\|H(u(\cdot, 0))\|_{L^{2}(\Omega)}$.

Proof. The second and third of these relations are a consequence of the horizontal line analysis of [17]. Thus, (2.4ii) follows from Lemma 3.5 of [17]; we use the fact that $H_{\varepsilon}^{\prime}(\xi) \geqslant \lambda$. In a similar way, (2.4iii) follows if we use the bound $H_{\varepsilon}^{\prime}(\xi) \leqslant \gamma / \varepsilon$ and otherwise use the proof of Lemma 3.5 . Finally, $(2.4 i, i v)$ may be proved as follows. Integrate (2.1i) against $H_{\varepsilon}\left(u^{\varepsilon}\right)$ to obtain

$$
\frac{1}{2} \frac{d}{d t}\left\|H_{\varepsilon}\left(u^{\varepsilon}\right)\right\|_{L^{2}(\Omega)}^{2}+\left(\nabla u^{\varepsilon}, \nabla H_{\varepsilon}\left(u^{\varepsilon}\right)\right)_{L^{2}(\Omega)}=-\left(H_{\varepsilon}\left(u^{\varepsilon}\right), f\left(u^{\varepsilon}\right)\right)_{L^{2}(\Omega)} .
$$

Integration in time yields (2.4i) via the Gronwall inequality and

$$
\left\|\sqrt{H_{\varepsilon}^{\prime}\left(u^{\varepsilon}\right)} \nabla u^{\varepsilon}\right\|_{L^{2}\left(0, T_{0} ; L^{2}(\Omega)\right)}^{2} \leqslant C,
$$

so that

$$
\begin{aligned}
& \left\|\nabla H_{\varepsilon}\left(u^{\varepsilon}\right)\right\|_{L^{2}\left(0, T_{0} ; L^{2}(\Omega)\right)}^{2} \\
& \quad \leqslant\left\|H_{\varepsilon}^{\prime}\left(u^{\varepsilon}\right)\right\|_{L^{\infty}\left(0, T_{0} ; L^{\infty}(\Omega)\right)}\left\|\sqrt{H_{\varepsilon}^{\prime}\left(u^{\varepsilon}\right)} \nabla u^{\varepsilon}\right\|_{L^{2}\left(0, T_{0} ; L^{2}(\Omega)\right)}^{2} \leqslant C / \varepsilon .
\end{aligned}
$$

Remark 2.2. Estimates (2.4ii, iii) follow formally by multiplying (2.1i) by $u_{t}^{\varepsilon}$ and integrating by parts to obtain

$$
\left(\frac{\partial}{\partial t} H_{\varepsilon}\left(u^{\varepsilon}\right), u_{t}^{\varepsilon}\right)_{L^{2}(\Omega)}+\frac{1}{2} \frac{d}{d t}\left\|\nabla u^{\varepsilon}\right\|_{L^{2}(\Omega)}^{2}+\left(f\left(u^{\varepsilon}\right), u_{t}^{\varepsilon}\right)_{L^{2}(\Omega)}=0 .
$$

From this, (2.4ii, iii) follow after integration in time and an application of Gronwall's inequality. This is purely formal, however, since it is not known that $u_{t}^{\varepsilon} \in H^{1}(\Omega)$. 
Proposition 2.2. There is a constant $C$, independent of $\varepsilon$, such that

$$
\left\|\left[H_{\varepsilon}\left(u^{\varepsilon}\right)\right]_{t}\right\|_{L^{\infty}\left(0, T_{0} ; L^{\prime}(\Omega)\right)} \leqslant C
$$

for $0<\varepsilon \leqslant \varepsilon_{0}$. In particular, $\partial H(u)(\cdot, t) / \partial t$ is a finite regular Baire measure $\mu_{t}$ on $\bar{\Omega}$, i.e.,

$$
\left\langle\psi, \frac{\partial H(u)}{\partial t}\right\rangle=\int_{\bar{\Omega}} \psi d \mu_{t}
$$

for all $\psi \in C(\bar{\Omega})$ and almost all $t \in\left[0, T_{0}\right]$ with $\operatorname{Ess~sup}_{t}\left\|\mu_{t}\right\|_{M(\bar{\Omega})} \leqslant C$ in the total variation norm and is the weak-* limit in this norm of $\left[H_{\varepsilon}\left(u^{\varepsilon}\right)\right]_{t}$.

Proof. The technique used in establishing (2.6) is an auxiliary equation argument due to Kruzhkov [22]. If $\tilde{u}^{\varepsilon}$ is a solution of (2.1i) with initial function in a fixed ball centered at $H(u(\cdot, 0))$ in $L^{2}(\Omega)$, we have

$$
\left[H_{\varepsilon}\left(u^{\varepsilon}\right)-H_{\varepsilon}\left(\tilde{u}^{\varepsilon}\right)\right]_{t}=\Delta\left(u^{\varepsilon}-\tilde{u}^{\varepsilon}\right)-\left[f\left(u^{\varepsilon}\right)-f\left(\tilde{u}^{\varepsilon}\right)\right] .
$$

Fixing $t_{0}, 0<t_{0} \leqslant T_{0}$, we define $\zeta=\zeta_{\varepsilon}$ to be the solution of the (backward) linear parabolic boundary value problem,

$$
\begin{array}{ll}
\text { (i) } h(x, t) \frac{\partial \zeta}{\partial t}=-\Delta \zeta & \text { in } D_{t_{0}}=\Omega \times\left[0, t_{0}\right), \\
\text { (ii) } \frac{\partial \zeta}{\partial \nu}=0 & \text { on } \partial \Omega \times\left[0, t_{0}\right), \\
\text { (iii) } \zeta\left(\cdot, t_{0}\right)=\operatorname{sgn}\left(u^{\varepsilon}-\tilde{u}^{\varepsilon}\right)\left(\cdot, t_{0}\right),
\end{array}
$$

where

$$
h(x, t)=\left(\frac{H_{\varepsilon}\left(u^{\varepsilon}\right)-H_{\varepsilon}\left(\tilde{u}^{\varepsilon}\right)}{u^{\varepsilon}-\tilde{u}^{\varepsilon}}\right)(x, t) \geqslant \lambda>0 .
$$

We note that a bounded solution $\zeta$ of (2.9) exists in the regularity class (2.2) satisfying

$$
\|\zeta\|_{L^{\infty}\left(D_{t_{0}}\right)} \leqslant 1 .
$$

The solution may be constructed by use of evolution operators as in Kato [21]. In this construction, the parabolic problems

$$
h\left(x, t_{*}\right) \frac{\partial \zeta_{*}}{\partial t}=-\Delta \zeta_{*}
$$

where $t_{*}$ is a fixed point in $\left(0, t_{0}\right)$, generate semigroups whose step-function sequences converge to the evolution operators. One can verify the maximum principle

$$
\left\|\zeta_{*}\right\|_{L^{\infty}\left(D_{t_{0}}\right)} \leqslant\left\|\operatorname{sgn}\left(u^{\varepsilon}-\tilde{u}^{\varepsilon}\right)\left(\cdot, t_{0}\right)\right\|_{L^{\infty}(\Omega)} \leqslant 1
$$

for the solution of (2.12), (2.9ii, iii) and this yields (2.11). We now have, for $v=u^{\varepsilon}-\tilde{u}^{\varepsilon}$,

$$
\begin{aligned}
{\left[\left(H_{\varepsilon}\left(u^{\varepsilon}\right)\right.\right.} & \left.\left.-H_{\varepsilon}\left(\tilde{u}^{\varepsilon}\right), \zeta\right)_{L^{2}(\Omega)}\right]_{0}^{t_{0}} \\
& =\int_{0}^{t_{0}}\left(h(x, t) \zeta_{t}, v\right)_{L^{2}(\Omega)} d t+\int_{0}^{t_{0}}(\nabla \zeta, \nabla v)_{L^{2}(\Omega)} \\
& =-\int_{0}^{t_{0}}\left(f\left(u^{\varepsilon}\right)-f\left(\tilde{u}^{\varepsilon}\right), \zeta\right)_{L^{2}(\Omega)} d t
\end{aligned}
$$


where we have used (2.8) and (2.9i). It follows from (2.11) and (2.14) that

$$
\begin{aligned}
& \left\|\left(H_{\varepsilon}\left(u^{\varepsilon}\right)-H_{\varepsilon}\left(\tilde{u}^{\varepsilon}\right)\right)\left(t_{0}\right)\right\|_{L^{1}(\Omega)} \\
& \quad \leqslant\left\|\left(H_{\varepsilon}\left(u^{\varepsilon}\right)-H_{\varepsilon}\left(\tilde{u}^{\varepsilon}\right)\right)(0)\right\|_{L^{\prime}(\Omega)}+C \int_{0}^{t_{0}}\left\|u^{\varepsilon}-\tilde{u}^{\varepsilon}\right\|_{L^{\prime}(\Omega)} d t .
\end{aligned}
$$

We now define, for fixed $\Delta t>0$,

$$
\tilde{u}^{\varepsilon}(x, t)=u^{\varepsilon}(x, t+\Delta t), \quad t \leqslant T_{0}-\Delta t .
$$

With this substitution in (2.15) we have, after dividing by $\Delta t$ and taking the supremum over $t_{0} \in\left[0, T_{0}-\Delta t\right]$,

$$
\begin{gathered}
\left\|\frac{H_{\varepsilon}\left(u^{\varepsilon}\right)(\cdot, t+\Delta t)-H_{\varepsilon}\left(u^{\varepsilon}\right)(\cdot, t)}{\Delta t}\right\|_{L^{\infty}\left(0, T_{0}-\Delta t ; L^{\prime}(\Omega)\right)} \\
\leqslant\left\|\frac{H_{\varepsilon}\left(u^{\varepsilon}\right)(\cdot, \Delta t)-H_{\varepsilon}\left(u^{\varepsilon}\right)(\cdot, 0)}{\Delta t}\right\|_{L^{\prime}(\Omega)} \\
+\int_{0}^{T_{0}-\Delta t}\left\|\frac{u^{\varepsilon}(\cdot, s+\Delta t)-u^{\varepsilon}(\cdot, s)}{\Delta t}\right\|_{L^{\prime}(\Omega)} d s .
\end{gathered}
$$

The second term on the right-hand side of (2.17) presents no difficulty as $\Delta t \rightarrow 0$. Indeed, it approaches the limit $\int_{0}^{T_{0}}\left\|u_{t}^{\varepsilon}\right\|_{L^{1}(\Omega)} d s$ which is clearly bounded above independently of $\varepsilon$ by (2.4ii). The first term on the right-hand side is more delicate. This we estimate by the method of lines which is a rigorous substitute for the continuity of (2.1i) at $t=0$. It is clearly enough to show that

$$
\frac{1}{\Delta t} \int_{0}^{\Delta t}\left\|\left[H_{\varepsilon}\left(u^{\varepsilon}\right)\right]_{t}\right\|_{L^{\prime}(\Omega)} d t \leqslant C .
$$

Thus, for given $\Delta t^{\prime} \leqslant \Delta t$, we consider the horizontal level approximations $u_{n}^{\varepsilon}$ defined by, for $N=\left[\Delta t / \Delta t^{\prime}+1\right]$,

$$
\frac{H_{\varepsilon}\left(u_{n}^{\varepsilon}\right)-H_{\varepsilon}\left(u_{n-1}^{\varepsilon}\right)}{\Delta t^{\prime}}-\Delta\left(u_{n}^{\varepsilon}-u_{n-1}^{\varepsilon}\right)=\Delta u_{n-1}^{\varepsilon}-f\left(u_{n}^{\varepsilon}\right), \quad 1 \leqslant n \leqslant N,
$$

subject to a homogeneous Neumann boundary condition on $\partial \Omega$. The weak form of (2.19) is adequate. Standard convergence arguments show that (2.18) follows from

$$
\sum_{n=1}^{N}\left\|\frac{H_{\varepsilon}\left(u_{n}^{\varepsilon}\right)-H_{\varepsilon}\left(u_{n-1}^{\varepsilon}\right)}{\Delta t^{\prime}}\right\|_{L^{\prime}(\Omega)} \Delta t^{\prime} \leqslant C \Delta t,
$$

together with the known weak convergence in $L^{2}\left(0, \Delta t ; L^{2}(\Omega)\right)$ of the step-function sequence of difference quotients to $\partial H_{\varepsilon}\left(u^{\varepsilon}\right) / \partial t$. By employing a semidiscrete version of the technique leading to (2.17), we see that, for $n=0, \ldots, N-1$, with $u_{0}^{\varepsilon}=u_{0}$,

$$
\begin{aligned}
& \left\|\frac{H_{\varepsilon}\left(u_{n+1}^{\varepsilon}\right)-H_{\varepsilon}\left(u_{n}^{\varepsilon}\right)}{\Delta t^{\prime}}\right\|_{L^{\prime}(\Omega)} \\
& \quad \leqslant\left\|\frac{H_{\varepsilon}\left(u_{1}^{\varepsilon}\right)-H_{\varepsilon}\left(u_{0}^{\varepsilon}\right)}{\Delta t^{\prime}}\right\|_{L^{\prime}(\Omega)}+C \sum_{k=1}^{n}\left\|u_{k+1}^{\varepsilon}-u_{k}^{\varepsilon}\right\|_{L^{\prime}(\Omega)} \\
& \quad \leqslant\left\|\frac{H_{\varepsilon}\left(u_{1}^{\varepsilon}\right)-H_{\varepsilon}\left(u_{0}^{\varepsilon}\right)}{\Delta t^{\prime}}\right\|_{L^{\prime}(\Omega)}+N C \Delta t^{\prime}+C|\Omega| \sum_{k=1}^{n} \frac{\left\|u_{k+1}^{\varepsilon}-u_{k}^{\varepsilon}\right\|_{L^{2}(\Omega)}^{2}}{\Delta t^{\prime}} \\
& \quad \leqslant\left\|\frac{H_{\varepsilon}\left(u_{1}^{\varepsilon}\right)-H_{\varepsilon}\left(u_{0}^{\varepsilon}\right)}{\Delta t^{\prime}}\right\|_{L^{\prime}(\Omega)}+C,
\end{aligned}
$$


by a discrete analogue of (2.4ii), so that the verification of $(2.20)$ reduces to the inequality

$$
\left\|\frac{H_{\varepsilon}\left(u_{1}^{\varepsilon}\right)-H_{\varepsilon}\left(u_{0}^{\varepsilon}\right)}{\Delta t^{\prime}}\right\|_{L^{\prime}(\Omega)} \leqslant C .
$$

Now fix a number $\delta \geqslant 1$, and for $g \in L^{1}(\Omega)$ set $S_{\delta}^{-} g=-S_{\delta}^{+}(-g)$, where

$$
S_{\delta}^{+} g(x)= \begin{cases}g(x), & g(x) \geqslant 1 / \delta \\ 1 / \delta, & \text { otherwise }\end{cases}
$$

Fix $p>1$ and, for $v \in L^{p}(\Omega), v \neq 0$, set $I_{p} \circ v=v|v|^{p-2} /\|v\|_{L^{p}(\Omega)}^{p^{-1}}$. Finally, for $0 \neq v \in H^{1}(\Omega)$ and $1<p \leqslant 2$, set $W_{p, \delta}^{ \pm}(v ; x)=\left(I_{p} \circ S_{\delta}^{ \pm}(v)\right)(x)$, and note that $W_{p, \delta}^{ \pm} \in H^{1}(\Omega)$. Now the following facts are evident: (Note the monotonicity of $I_{p} \circ S_{\delta}^{ \pm}$.)

$$
\begin{gathered}
v \in L^{2}(\Omega) \Rightarrow I_{q} \circ v \rightarrow \operatorname{sgn} v\left(\text { dominated by a function in } L^{2}(\Omega)\right), \quad q \searrow 1, \\
\nabla v \cdot \nabla W_{p, \delta}^{ \pm}(v ; \cdot) \geqslant 0, \quad\left\|I_{p} \circ v\right\|_{L^{p^{\prime}(\Omega)}}=1, \quad 1 / p+1 / p^{\prime}=1 .
\end{gathered}
$$

To verify (2.21), select $v=u_{1}^{\varepsilon}-u_{0}^{\varepsilon}$ and multiply (2.19), for $n=1$, by $W_{p, \delta}^{ \pm}(v ; \cdot)$. Integrate over $\Omega$, neglect the energy term and let $\delta \rightarrow \infty$. We obtain, following two applications of Hölder's inequality,

$$
\frac{1}{\Delta t^{\prime}} \int_{\Omega}\left[H_{\varepsilon}\left(u_{1}^{\varepsilon}\right)-H_{\varepsilon}\left(u_{0}^{\varepsilon}\right)\right] \cdot I_{p} \circ v^{ \pm} \leqslant\left\|\Delta u_{0}\right\|_{L^{p}(\Omega)}+C\left\|u_{1}^{\varepsilon}\right\|_{L^{p}(\Omega)} .
$$

Here we have assumed for the moment that $\Delta u_{0} \in L^{2}(\Omega)$. (2.21) now follows by multiplying this inequality by $|\Omega|^{-1 / p}$, letting $p \searrow 1$, then cancelling $|\Omega|^{-1}$ to obtain

$$
\left\|\frac{\left[H_{\varepsilon}\left(u_{1}^{\varepsilon}\right)-H_{\varepsilon}\left(u_{0}^{\varepsilon}\right)\right]^{ \pm}}{\Delta t^{\prime}}\right\|_{L^{\prime}(\Omega)} \leqslant\left\|\Delta u_{0}\right\|_{L^{\prime}(\Omega)}+C\left\|u_{1}^{\varepsilon}\right\|_{L^{\prime}(\Omega)} .
$$

The right-hand side is bounded by the hypothesis on $u_{0}$ and estimates on $u_{1}^{\varepsilon}$ derived in [17]. Note that we have applied the Lebesgue dominated convergence theorem and the fact that $u_{1}^{\varepsilon}-u_{0}^{\varepsilon}$ and $H_{\varepsilon}\left(u_{1}^{\varepsilon}\right)-H_{\varepsilon}\left(u_{0}^{\varepsilon}\right)$ have the same sign. The boundedness of the right-hand side of (2.17) independent of $\varepsilon$ is sufficient to insure (2.6), since almost every $t$ is a Lebesgue point (cf. Hille and Phillips [16, p. 88]).

This completes the proof under the asumption, say, that $u_{0} \in H^{2}(\Omega)$. A standard smoothing argument completes the proof under the hypothesis $u_{0} \in W^{2,1}(\Omega)$ stated in the introduction.

The final statement follows from the isometric inclusion of $L^{1}(\Omega)$ in $M(\bar{\Omega})$ and the weak-* compactness of the latter [14] and the standard identification of the limit.

Remark 2.3. By inequality (3.3), to follow, we see that the proof of the preceding proposition yields the estimate

$$
\left\|u_{t}^{\varepsilon}\right\|_{L^{\infty}\left(0, T ; L^{1}(\Omega)\right)} \leqslant C
$$

for some constant $C$ independent of $\varepsilon$.

The following result is a strengthening of (2.4i). 
Proposition 2.3. There is a constant $C$ independent of $\varepsilon$ such that

$$
\begin{gathered}
\left\|H_{\varepsilon}\left(u^{\varepsilon}\right)\right\|_{L^{\infty}\left(0, T_{0} ; L^{\infty}(\Omega)\right)} \leqslant C, \\
\left\|u^{\varepsilon}\right\|_{L^{\infty}\left(0, T_{0} ; L^{\infty}(\Omega)\right)} \leqslant C / \lambda .
\end{gathered}
$$

Proof. We sketch a proof based on the method of horizontal lines where $f$ is evaluated explicitly. Thus, we consider the semidiscretization, for $\Delta t=T_{0} / M$, $n=0,1, \ldots, M-1$,

$$
\begin{array}{ll}
\text { (i) } \frac{H_{\varepsilon}\left(u_{n+1}^{\varepsilon}\right)-H_{\varepsilon}\left(u_{n}^{\varepsilon}\right)}{\Delta t}-\Delta u_{n+1}^{\varepsilon}+f\left(u_{n}^{\varepsilon}\right)=0 & \text { in } \Omega, \\
\text { (ii) } \frac{\partial u_{n+1}^{\varepsilon}=0}{\partial \nu}=0 & \text { on } \partial \Omega,
\end{array}
$$

of recursively defined nonlinear Neumann problems corresponding to the time levels $t_{n}=(n+1) \Delta t$ with $u_{0}^{\varepsilon}=u_{0}$. One requires that (2.24) hold in weak form. By known convergence properties of the horizontal line method it is enough to prove

$$
\left\|H_{\varepsilon}\left(u_{n}^{\varepsilon}\right)\right\|_{L^{\infty}(\Omega)} \leqslant C
$$

where $C$ is independent of $n, \Delta t$, and $\varepsilon$. In fact, we shall prove

$$
\left\|H_{\varepsilon}\left(u_{n}^{\varepsilon}\right)\right\|_{L^{\infty}(\Omega)} \leqslant C\left\{\left\|H_{\varepsilon}\left(u_{0}^{\varepsilon}\right)\right\|_{L^{\infty}(\Omega)}+\sum_{i=0}^{n-1}\left\|u_{i}^{\varepsilon}\right\|_{L^{\infty}(\Omega)} \Delta t\right\}
$$

for each $n=1, \ldots, M$, which implies (2.25) if we use the facts that $u_{0} \in L^{\infty}(\Omega)$, that $H_{\varepsilon}\left(u_{0}\right)$ can be estimated in terms of $\left\|u_{0}\right\|_{L^{\infty}}$, and

$$
\left\|u_{i}^{\varepsilon}\right\|_{L^{\infty}(\Omega)}=\left\|J_{\varepsilon} \circ H_{\varepsilon}\left(u_{i}^{\varepsilon}\right)\right\|_{L^{\infty}(\Omega)} \leqslant \frac{1}{\lambda}\left\|H_{\varepsilon}\left(u_{i}^{\varepsilon}\right)\right\|_{L^{\infty}(\Omega)}
$$

in (2.26) followed by the discrete Gronwall inequality. Now fix $j$ to be an arbitrary integer, and define the truncation operators, required to obtain suitable $H^{1}$ test functions in the weak formulation, by

$$
\Theta_{j, l} \circ v= \begin{cases}{[v(x)]^{l},} & |v(x)| \leqslant j, \\ {[\operatorname{sgn} v(x) j]^{l},} & |v(x)|>j,\end{cases}
$$

for $l \geqslant 1$. For $p$ an arbitrary even integer,

$$
v=\Theta_{j, p-1} \circ H_{\varepsilon}\left(u_{n}^{\varepsilon}\right) \in H^{1}(\Omega),
$$

and this choice of a test function in the weak form of (2.24) yields

$$
\begin{gathered}
\int_{\Omega} v^{p}+\Delta t(p-1) \int_{\left\{\left|u_{n}^{\varepsilon}\right| \leqslant j\right\}}\left|\nabla u_{n}^{\varepsilon}\right|^{2} H_{\varepsilon}^{\prime}\left(u_{n}^{\varepsilon}\right)\left[H_{\varepsilon}\left(u_{n}^{\varepsilon}\right)\right]^{p-2} d x \\
\leqslant \frac{1}{q} \int_{\Omega} v^{p}+\frac{1}{p} \int_{\Omega}\left|H_{\varepsilon}\left(u_{n-1}^{\varepsilon}\right)-f\left(u_{n-1}^{\varepsilon}\right) \Delta t\right|^{p}
\end{gathered}
$$

where we have used the inequality

$$
a b \leqslant \frac{1}{p} a^{p}+\frac{1}{q} b^{q}, \quad a \geqslant 0, b \geqslant 0, \frac{1}{p}+\frac{1}{q}=1,
$$


with $b=v, a=H_{\varepsilon}\left(u_{n}^{\varepsilon}\right)-f\left(u_{n-1}^{\varepsilon}\right) \Delta t$. Neglecting the energy term in (2.29), multiplying through by $p$, taking $p$ th roots and letting $p \rightarrow \infty$, followed by $j \rightarrow \infty$, gives the inequality

$$
\left\|H_{\varepsilon}\left(u_{n}^{\varepsilon}\right)\right\|_{L^{\infty}(\Omega)} \leqslant\left\|H_{\varepsilon}\left(u_{n-1}^{\varepsilon}\right)\right\|_{L^{\infty}(\Omega)}+\left\|f\left(u_{n-1}^{\varepsilon}\right)\right\|_{L^{\infty}(\Omega)} \Delta t .
$$

(2.26) is now immediate from the Lipschitz property of $f$. We have thus established (2.23i). The bound (2.23ii) is now immediate, and by the continuity of $u^{\varepsilon}$ and $H_{\varepsilon}\left(u^{\varepsilon}\right)$ we conclude in fact that the bounds hold for all $0<t<T_{0}$.

Remark 2.4. The preceding two propositions enable us to conclude that

$$
\left\|H_{\varepsilon}\left(u^{\varepsilon}\right)\right\|_{L^{\infty}\left(0, T_{0} ; H^{\prime}(\Omega)\right)} \leqslant \frac{C}{\sqrt{\varepsilon}} .
$$

We need only a gradient estimate to verify (2.31). Integrate (2.1i) against $H_{\varepsilon}\left(u^{\varepsilon}\right)$ to see that

$$
\begin{aligned}
& \left(\nabla u^{\varepsilon}, \nabla H_{\varepsilon}\left(u^{\varepsilon}\right)\right)_{L^{2}(\Omega)}=-\left(H_{\varepsilon}\left(u^{\varepsilon}\right)_{t}+f\left(u^{\varepsilon}\right), H_{\varepsilon}\left(u^{\varepsilon}\right)\right)_{L^{2}(\Omega)} \\
& \quad \leqslant\left(\left\|\left[H_{\varepsilon}\left(u^{\varepsilon}\right)\right]_{t}\right\|_{L^{\infty}\left(0, T_{0} ; L^{1}(\Omega)\right)}+C\left\|u^{\varepsilon}\right\|_{L^{\infty}(D)}\right)\left\|H_{\varepsilon}\left(u^{\varepsilon}\right)\right\|_{L^{\infty}\left(0, T_{0} ; L^{\infty}(\Omega)\right)} \\
& \quad \leqslant C .
\end{aligned}
$$

Since $H_{\varepsilon}^{\prime} \leqslant \gamma / \varepsilon$,

$$
\left\|\nabla H_{\varepsilon}\left(u^{\varepsilon}\right)\right\|_{L^{\infty}\left(0, T_{0} ; L^{2}(\Omega)\right)}^{2} \leqslant \frac{\gamma}{\varepsilon}\left\|\sqrt{H_{\varepsilon}^{\prime}\left(u^{\varepsilon}\right)} \nabla u^{\varepsilon}\right\|_{L^{\infty}\left(0, T_{0} ; L^{2}(\Omega)\right)}^{2} \leqslant \frac{C}{\varepsilon},
$$

and (2.31) follows.

3. Uniqueness Estimates and Convergence of the Regularization. We begin by establishing an a priori estimate for solutions of (1.8) which implies a uniqueness result (cf. also Damlamian [11]).

Proposition 3.1. The solution of (1.8) is unique within the class $\mathcal{C}$.

Proof. Let $u$ and $w$ be solutions in $\mathcal{C}$ of (1.8). Subtracting relation (1.13), satisfied by $w$ from the corresponding relation satisfied by $u$, we have, after multiplication by $H(u)-H(w)$ and integration over $\Omega$,

$$
\begin{aligned}
& \left(\frac{\partial}{\partial t} T[H(u)-H(w)], H(u)-H(w)\right)_{L^{2}(\Omega)}+(u-w, H(u)-H(w))_{L^{2}(\Omega)} \\
& +(T[f(u)-f(w)], H(u)-H(w))_{L^{2}(\Omega)}=\frac{1}{|\Omega|} j(u-w) j(H(u)-H(w))
\end{aligned}
$$

a relation which holds for $0<t \leqslant T_{0}$. From (1.11) it follows that

$$
\left(\frac{\partial}{\partial t} T[H(u)-H(w)], H(u)-H(w)\right)_{L^{2}(\Omega)}=\frac{1}{2} \frac{d}{d t}\|H(u)-H(w)\|_{F}^{2},
$$


and substitution of this relation into (3.1), followed by integration in time, yields

$$
\begin{aligned}
& \frac{1}{2}\|H(u)-H(w)\|_{F}^{2}+\int_{0}^{t}(u-w, H(u)-H(w))_{L^{2}(\Omega)} d \tau \\
& \quad+\int_{0}^{t}(f(u)-f(w), T[H(u)-H(w)])_{L^{2}(\Omega)} d \tau \\
& =\int_{0}^{t}\left(\frac{1}{|\Omega|} \int_{\Omega}(u-w) \int_{\Omega}[H(u)-H(w)]\right) d \tau+\frac{1}{2}\left\|H\left(u_{0}\right)-H\left(w_{0}\right)\right\|_{F}^{2}
\end{aligned}
$$

for all $0<t \leqslant T_{0}$. By (1.5i) we have the inequality

$$
[H(a)-H(b)](a-b) \geqslant \lambda(a-b)^{2}, \quad a, b \in \mathbf{R},
$$

so that the second term in (3.2) is nonnegative. Direct estimation and the CauchySchwarz inequality yield

$$
\begin{gathered}
\left(\frac{1}{|\Omega|} \int_{\Omega}[H(u)-H(w)]\right)^{2} \leqslant\|H(u)-H(w)\|_{F}^{2}, \\
(f(u)-f(w), T[H(u)-H(w)])_{L^{2}(\Omega)} \leqslant\|f(u)-f(w)\|_{F}\|H(u)-H(w)\|_{F} .
\end{gathered}
$$

The right-hand side of (3.2) is thus bounded from above by

for arbitrary $\eta>0$, and the right-hand side of (3.5) is bounded above by

$$
\frac{1}{2}\left\{\eta C\|u-w\|_{L^{2}(\Omega)}^{2}+\eta^{-1}\|H(u)-H(w)\|_{F}^{2}\right\}
$$

where we have made use of the continuous injection of $L^{2}(\Omega)$ into $F$ and the Lipschitz property of $f$. It is now clear upon use of (3.2)-(3.5) and the bounds (3.6), (3.7) that there exists $\eta>0$ such that, for $0<t \leqslant T_{0}$,

$$
\begin{aligned}
\frac{1}{2}\|H(u)-H(w)\|_{F}^{2} & +\frac{\lambda}{2} \int_{0}^{t}\|u-w\|_{L^{2}(\Omega)}^{2} d \tau \\
& \leqslant \eta^{-1} \int_{0}^{t}\|H(u)-H(w)\|_{F}^{2} d \tau+\frac{1}{2}\left\|H\left(u_{0}\right)-H\left(w_{0}\right)\right\|_{F}^{2}
\end{aligned}
$$

By Gronwall's inequality,

(3.8ii) $\|H(u)-H(w)\|_{L^{\infty}\left(0, T_{0} ; F\right)}^{2}+\|u-w\|_{L^{2}\left(0, T_{0} ; L^{2}(\Omega)\right)}^{2} \leqslant C\left\|H\left(u_{0}\right)-H\left(w_{0}\right)\right\|_{F}^{2}$.

Thus, $T H\left(u_{0}\right)=T H\left(w_{0}\right)$ implies $H(u)=H(w)$ and $u=w, 0<t \leqslant T_{0}$.

We shall need to make an additional assumption* concerning the initial function $u_{0}(x)$ :

$$
\left|\left\{x \in \Omega: 0 \leqslant u_{0}(x) \leqslant \varepsilon\right\}\right| \leqslant C \varepsilon, \quad 0<\varepsilon \leqslant \varepsilon_{0} .
$$

Remark 3.1. Assumption (3.9) is unnecessary if we define the initial data for the regularized problem to be

$$
H_{\varepsilon}\left(u^{\varepsilon}(x, 0)\right)=H\left(u_{0}(x)\right) \text { on } \Omega .
$$

*Strict inequality at 0 would be preferred. This is possible if $H\left(u_{0}\right)$ vanishes a.e. when $u_{0}$ vanishes. 
However, the choice we made in (2.1iii)

$$
u^{\varepsilon}(x, 0)=u_{0}(x) \text { on } \Omega,
$$

is more natural. Although it would suffice to assume the right side of (3.9) is only $O(\sqrt{\varepsilon})$ for some of our results, (3.9) is not at all restrictive.

We establish convergence rates for the regularization in the following

THEOREM 3.2. Let $u$ be the solution of (1.8), and let $u^{\varepsilon}$ be the solution of the regularized problem (2.1). Then there exists a constant $C$ such that the estimates

(i) $\left\|H_{\varepsilon}\left(u^{\varepsilon}\right)-H(u)\right\|_{L^{\infty}\left(0, T_{0} ; F\right)} \leqslant C \sqrt{\varepsilon}$,

(ii) $\left\|u^{\varepsilon}-u\right\|_{L^{2}(D)} \leqslant C \sqrt{\varepsilon}$,

hold for $0<\varepsilon \leqslant \varepsilon_{0}$.

Proof. We find it convenient to use the inverse formulation, with

$$
J=H^{-1}, \quad J_{\varepsilon}=H_{\varepsilon}^{-1}, \quad v=H(u) \text { and } v^{\varepsilon}=H_{\varepsilon}\left(u^{\varepsilon}\right) .
$$

Using (1.13) for both $u$ and $u^{\varepsilon}$, we have, after subtraction, multiplication by $v^{\varepsilon}-v$ and integration over $\Omega$,

$$
\begin{aligned}
\frac{1}{2} \frac{d}{d t} \| v^{\varepsilon}- & v \|_{F}^{2}+\left(J_{\varepsilon}\left(v^{\varepsilon}\right)-J_{\varepsilon}(v), v^{\varepsilon}-v\right)_{L^{2}(\Omega)} \\
= & \frac{1}{|\Omega|} j\left(J_{\varepsilon}\left(v^{\varepsilon}\right)-J(v)\right) j\left(v^{\varepsilon}-v\right)+\left(J(v)-J_{\varepsilon}(v), v^{\varepsilon}-v\right)_{L^{2}(\Omega)} \\
& -\left(T\left[f \circ J_{\varepsilon}\left(v^{\varepsilon}\right)-f \circ J(v)\right], v^{\varepsilon}-v\right)_{L^{2}(\Omega)}
\end{aligned}
$$

The right side of (3.11) may be bounded by

$$
C\left\|v^{\varepsilon}-v\right\|_{F}^{2}+\frac{\lambda}{4}\left\|J_{\varepsilon}\left(v^{\varepsilon}\right)-J_{\varepsilon}(v)\right\|_{L^{2}(\Omega)}^{2}+C\left\|v^{\varepsilon}-v\right\|_{L^{2}(\Omega)}\left\|J_{\varepsilon}(v)-J(v)\right\|_{L^{2}(\Omega)},
$$

where we have used the arguments used to derive (3.8).

The third term in (3.12) is estimated by

$$
\begin{aligned}
C\left\|v^{\varepsilon}-v\right\|_{L^{2}(\Omega)}\left\|J_{\varepsilon}(v)-J(v)\right\|_{L^{2}(\Omega)} \\
\quad \leqslant \frac{\varepsilon}{4 \gamma}\left\|v^{\varepsilon}-v\right\|_{L^{2}(\Omega)}^{2}+\left(C^{\prime} / \varepsilon\right)\left\|J_{\varepsilon}(v)-J(v)\right\|_{L^{2}(\Omega)}^{2} \\
\quad \leqslant \frac{\varepsilon}{4 \gamma}\left\|v^{\varepsilon}-v\right\|_{L^{2}(\Omega)}^{2}+C^{\prime \prime} \varepsilon
\end{aligned}
$$

since by the definition of $J$ and $J_{\varepsilon}$

$$
\left\|J_{\varepsilon}(v)-J(v)\right\|_{L^{\infty}\left(0, T_{0} ; L^{\infty}(\Omega)\right)} \leqslant C \varepsilon .
$$

Using (1.18) and (4.21), respectively, we can absorb into the left-hand side of (3.11) the second term in (3.12) and the first term on the right-hand side of (3.13). We get

$$
\frac{1}{2} \frac{d}{d t}\left\|v^{\varepsilon}-v\right\|_{F}^{2}+\frac{1}{2}\left(J_{\varepsilon}\left(v^{\varepsilon}\right)-J_{\varepsilon}(v), v^{\varepsilon}-v\right)_{L^{2}(\Omega)} \leqslant C\left[\varepsilon+\left\|v^{\varepsilon}-v\right\|_{F}^{2}\right]
$$


Bound (3.14) remains valid if $J_{\varepsilon}(v)$ is replaced by $J(v)$ in the second term on the left. Integrate in time and apply Gronwall's inequality to obtain

$$
\begin{aligned}
& \left\|v^{\varepsilon}-v\right\|_{L^{\infty}\left(0, T_{0} ; F\right)}^{2}+\int_{0}^{T_{0}}\left(J_{\varepsilon}\left(v^{\varepsilon}\right)-J(v), v^{\varepsilon}-v\right)_{L^{2}(\Omega)} d t \\
& \quad=\left\|H_{\varepsilon}\left(u^{\varepsilon}\right)-H(u)\right\|_{L^{\infty}\left(0, T_{0} ; F\right)}^{2}+\int_{0}^{T_{0}}\left(H_{\varepsilon}\left(u^{\varepsilon}\right)-H(u), u^{\varepsilon}-u\right)_{L^{2}(\Omega)} d t \\
& \quad \leqslant C\left[\varepsilon+\left\|H_{\varepsilon}\left(u_{0}\right)-H\left(u_{0}\right)\right\|_{F}^{2}\right] .
\end{aligned}
$$

The inequality (cf. (3.18))

$$
\left(H_{\varepsilon}\left(u^{\varepsilon}\right)-H(u), u^{\varepsilon}-u\right)_{L^{2}(\Omega)} \geqslant \lambda\left\|u^{\varepsilon}-u\right\|_{L^{2}(\Omega)}^{2}-C \varepsilon
$$

allows us to conclude that

$$
\begin{gathered}
\left\|H_{\varepsilon}\left(u^{\varepsilon}\right)-H(u)\right\|_{L^{\infty}\left(0, T_{0} ; F\right)}^{2}+\lambda\left\|u^{\varepsilon}-u\right\|_{L^{2}\left(0, T_{0} ; L^{2}(\Omega)\right)}^{2} \\
\leqslant C\left[\varepsilon+\left\|H_{\varepsilon}\left(u_{0}\right)-H\left(u_{0}\right)\right\|_{F}^{2}\right] .
\end{gathered}
$$

By (3.9),

$$
\begin{aligned}
\| H_{\varepsilon}\left(u_{0}\right)- & H\left(u_{0}\right)\left\|_{F}^{2} \leqslant C\right\| H_{\varepsilon}\left(u_{0}\right)-H\left(u_{0}\right) \|_{L^{2}(\Omega)}^{2} \\
& \leqslant C\left\|\left(H_{\varepsilon}-H\right)\left(u_{0}\right)\right\|_{L^{\infty}(\Omega)}^{2}\left|\left\{x \in \Omega: 0 \leqslant u_{0} \leqslant \varepsilon\right\}\right|+(\mu \varepsilon|\Omega|)^{2} \leqslant C \varepsilon
\end{aligned}
$$

so that (3.17) yields (3.10).

To verify (3.16) it suffices to show that

$$
\left(H_{\varepsilon}(a)-H(b)\right)(a-b) \geqslant \lambda(a-b)^{2}-C \varepsilon
$$

for any real $a$ and $b$. By (1.5i) and (1.18) the inequality is obvious unless both $a$ and $b$ are in $[0, \varepsilon]$. In the latter case

$$
\left|\left(H_{\varepsilon}(a)-H(b)\right)(a-b)\right| \leqslant(A+\mu \varepsilon) \cdot \varepsilon
$$

which completes the argument.

We shall also need regularization estimates for the discrete-time problem introduced in Section 5.

THeOREM 3.3. Let $M$ be a positive integer, let $\Delta t=T_{0} / M$, and define $t_{n}=n \cdot \Delta t$ for $n=0,1, \ldots, M$. Let $u^{n}$ denote $u\left(\cdot, t_{n}\right)$ and $u^{\varepsilon, n}$ denote $u^{\varepsilon}\left(\cdot, t_{n}\right)$, where $u$ and $u^{\varepsilon}$ are the solutions of (1.1) and (2.1), respectively. Then for some constant $\eta$

$$
\operatorname{Max}_{n}\left\|H_{\varepsilon}\left(u^{\varepsilon}\right)^{n}-H(u)^{n}\right\|_{F}^{2}+\eta \sum_{n=0}^{M}\left\|u^{\varepsilon, n}-u^{n}\right\|_{L^{2}(\Omega)}^{2} \cdot \Delta t \leqslant C\left(\frac{(\Delta t)^{2}}{\varepsilon}+\varepsilon\right),
$$

so that $u^{n}, H(u)^{n}$ are in $L^{\infty}(\Omega)$ with the bound of (2.23) and

$$
\operatorname{Max}_{n}\left\|H_{\varepsilon}\left(u^{\varepsilon}\right)^{n}-H(u)^{n}\right\|_{F}^{2}+\eta \sum_{n=0}^{M}\left\|u^{\varepsilon, n}-u^{n}\right\|_{L^{2}(\Omega)}^{2} \cdot \Delta t \leqslant C((\Delta t)+\varepsilon) .
$$


Proof. In the inverse formulation we have, from (1.13) and (2.3),

$$
\begin{aligned}
& \frac{T\left(v^{\varepsilon, n+1}-v^{\varepsilon, n}\right)}{\Delta t}+J_{\varepsilon}\left(v^{\varepsilon, n+1}\right)+T f \circ J_{\varepsilon}\left(v^{\varepsilon, n+1}\right) \\
& =-\frac{\partial}{\partial t} T v^{\varepsilon}\left(t_{n+1}\right)+\frac{T\left(v^{\varepsilon, n+1}-v^{\varepsilon, n}\right)}{\Delta t}+\frac{1}{|\Omega|} \int_{\Omega} J_{\varepsilon}\left(v^{\varepsilon, n+1}\right) \\
& =\frac{-1}{\Delta t} \int_{t_{n}}^{t_{n+1}}\left(\tau-t_{n}\right) \frac{\partial^{2}}{\partial t^{2}} T v^{\varepsilon}(\tau) d \tau+\frac{1}{|\Omega|} \int_{\Omega} J_{\varepsilon}\left(v^{\varepsilon, n+1}\right)
\end{aligned}
$$

and

$$
\begin{aligned}
& \frac{T\left(v^{n+1}-v^{n}\right)}{\Delta t}+J\left(v^{n+1}\right)+T f \circ J\left(v^{n+1}\right) \\
& \quad=\frac{-1}{\Delta t} \int_{t_{n}}^{t_{n+1}}\left(\tau-t_{n}\right) \frac{\partial^{2}}{\partial t^{2}} T v(\tau) d \tau+\frac{1}{|\Omega|} \int_{\Omega} J\left(v^{n+1}\right) .
\end{aligned}
$$

Subtract (3.21ii) from (3.2li) and integrate the difference against $\left(v^{\varepsilon, n+1}-v^{n+1}\right)$ to obtain

$$
\begin{aligned}
& \frac{1}{2 \Delta t}\left\{\left\|v^{\varepsilon, n+1}-v^{n+1}\right\|_{F}^{2}-\left\|v^{\varepsilon, n}-v^{n}\right\|_{F}^{2}\right\} \\
&+\left(J_{\varepsilon}\left(v^{\varepsilon, n+1}\right)-J_{\varepsilon}\left(v^{n+1}\right), v^{\varepsilon, n+1}-v^{n+1}\right)_{L^{2}(\Omega)} \\
& \leqslant-\left(f \circ J_{\varepsilon}\left(v^{\varepsilon, n+1}\right)-f \circ J_{\varepsilon}\left(v^{n+1}\right), v^{\varepsilon, n+1}-v^{n+1}\right)_{F} \\
&+\left(f \circ J\left(v^{n+1}\right)-f \circ J_{\varepsilon}\left(v^{n+1}\right), v^{\varepsilon, n+1}-v^{n+1}\right)_{F} \\
&+\left(J\left(v^{n+1}\right)-J_{\varepsilon}\left(v^{n+1}\right), v^{\varepsilon, n+1}-v^{n+1}\right)_{L^{2}(\Omega)} \\
&-\left(\frac{1}{\Delta t} \int_{t_{n}}^{t_{n+1}}\left(\tau-t_{n}\right)\left[T\left(v^{\varepsilon}(\tau)-v(\tau)\right)\right]_{t t} d \tau, v^{\varepsilon, n+1}-v^{n+1}\right)_{L^{2}(\Omega)} \\
&+\frac{1}{|\Omega|} \int_{\Omega}\left[J_{\varepsilon}\left(v^{\varepsilon, n+1}\right)-J\left(v^{n+1}\right)\right] \int_{\Omega}\left(v^{\varepsilon, n+1}-v^{n+1}\right) \\
&=(\mathrm{I})+(\mathrm{II})+(\mathrm{III})+(\mathrm{IV})+(\mathrm{V}) .
\end{aligned}
$$

As in (3.5)-(3.7), we may bound (I) by

$$
\frac{\lambda}{8}\left\|J_{\varepsilon}\left(v^{\varepsilon, n+1}\right)-J_{\varepsilon}\left(v^{n+1}\right)\right\|_{L^{2}(\Omega)}^{2}+C\left\|v^{\varepsilon, n+1}-v^{n+1}\right\|_{F}^{2} .
$$

Term (V) is bounded similarly. We may bound (II) and (III) by

$$
|(\mathrm{II})|+|(\mathrm{III})| \leqslant C \varepsilon+\frac{\varepsilon}{8 \gamma}\left\|v^{\varepsilon, n+1}-v^{n+1}\right\|_{L^{2}(\Omega)}^{2}
$$

by using a technique similar to that used in estimating (3.13).

To treat term (IV), notice that by (1.13) and (2.3)

$$
\begin{aligned}
{\left[T\left(v^{\varepsilon}-v\right)\right]_{t t}=} & -\left(\left[J_{\varepsilon}\left(v^{\varepsilon}\right)\right]_{t}-[J(v)]_{t}\right)-T\left[\left[f \circ J_{\varepsilon}\left(v^{\varepsilon}\right)\right]_{t}-[f \circ J(v)]_{t}\right] \\
& +\frac{1}{|\Omega|} \int_{\Omega}\left(v_{t}^{\varepsilon}-v_{t}\right),
\end{aligned}
$$


so that Propositions 2.1 and 2.2 and the previous equation imply that

$$
\begin{gathered}
\left\|\left[T\left(v^{\varepsilon}-v\right)\right]_{t}\right\|_{L^{2}\left(0, T_{0} ; L^{2}(\Omega)\right)} \leqslant C, \\
\left\|\left[T\left(v^{\varepsilon}-v\right)\right]_{t}\right\|_{L^{\infty}\left(0, T_{0} ; M(\bar{\Omega})\right)} \leqslant\left\|V_{t}^{\varepsilon}-V_{t}\right\|_{L^{\infty}\left(0, T_{0} ; M(\bar{\Omega})\right)} \leqslant C .
\end{gathered}
$$

Note that the second inequality in (3.25) requires the boundedness of $T$ as a mapping from $L^{1}(\Omega)$ into itself (cf. Brezis and Strauss [6, Lemma 23]) and approximation of $v_{t}$.

To prove (3.19), bound term (IV) by

$$
\begin{aligned}
\frac{1}{\Delta t} \int_{t_{n}}^{t_{n+1}}\left(\tau-t_{n}\right) & \left\|\left[T\left(v^{\varepsilon}-v\right)\right]_{t t}\right\|_{L^{2}(\Omega)} d \tau \cdot\left\|v^{\varepsilon, n+1}-v^{n+1}\right\| L_{L^{2}(\Omega)} \\
\leqslant & \sqrt{\Delta t} \cdot\left\|\left[T\left(v^{\varepsilon}-v\right)\right]_{t t}\right\|_{L^{2}\left(t_{n}, t_{n+1} ; L^{2}(\Omega)\right)} \cdot\left\|v^{\varepsilon, n+1}-v^{n+1}\right\| L_{L^{2}(\Omega)} \\
\leqslant & C\left(\frac{\Delta t}{\varepsilon}\left\|\left[T\left(v^{\varepsilon}-v\right)\right]_{t t}\right\|_{L^{2}\left(t_{n}, t_{n+1} ; L^{2}(\Omega)\right)}^{2}\right) \\
& +\frac{1}{8 \gamma} \varepsilon\left\|v^{\varepsilon, n+1}-v^{n+1}\right\|_{L^{2}(\Omega)}^{2}
\end{aligned}
$$

where the constant $\gamma$ was defined in (1.18); use (4.21) to absorb all terms of the form of the last term into the left side of (3.22) and (1.18) to absorb the first term of (3.23). Combine (3.22), (3.23), (3.24), and (3.26) to obtain

$$
\begin{aligned}
\frac{1}{2 \Delta t}\left\{\left\|v^{\varepsilon, n+1}-v^{n+1}\right\|_{F}^{2}-\left\|v^{\varepsilon, n}-v^{n}\right\|_{F}^{2}\right\} & \\
& +\frac{1}{2}\left(J_{\varepsilon}\left(v^{\varepsilon, n+1}\right)-J_{\varepsilon}\left(v^{n+1}\right), v^{\varepsilon, n+1}-v^{n+1}\right)_{L^{2}(\Omega)} \\
\leqslant & {\left[\varepsilon+\frac{\Delta t}{\varepsilon}\left\|\left[T\left(v^{\varepsilon}-v\right)\right]_{t t}\right\|_{L^{2}\left(t_{n}, t_{n+1} ; L^{2}(\Omega)\right)}^{2}+\left\|v^{\varepsilon, n+1}-v^{n+1}\right\|_{F}^{2}\right] . }
\end{aligned}
$$

Notice that we may replace the second term on the left side of (3.27) with

$$
\left(J_{\varepsilon}\left(v^{\varepsilon, n+1}\right)-J\left(v^{n+1}\right), v^{\varepsilon, n+1}-v^{n+1}\right)_{L^{2}(\Omega)} .
$$

Multiply the modified version of (3.27) by $\Delta t$, sum on $n$, and use the discrete version of Gronwall's lemma [17, Lemma 3.3, p. 251] to obtain

$$
\text { (3.28) } \begin{aligned}
\operatorname{Max}_{n}\left\|v^{\varepsilon, n+1}-v^{n+1}\right\|_{F}^{2} \\
\quad+\eta \sum_{n=0}^{M-1}\left(J_{\varepsilon}\left(v^{\varepsilon, n+1}\right)-J\left(v^{n+1}\right), v^{\varepsilon, n+1}-v^{n+1}\right)_{L^{2}(\Omega)} \cdot \Delta t \\
\leqslant C\left[\varepsilon+\left\|H_{\varepsilon}\left(u_{0}\right)-H\left(u_{0}\right)\right\|_{F}^{2}\right]+C \frac{(\Delta t)^{2}}{\varepsilon}\left\|\left[T\left(v^{\varepsilon}-v\right)\right]_{t t}\right\|_{L^{2}\left(0, T_{0} ; L^{2}(\Omega)\right)}^{2} \\
\leqslant C\left(\varepsilon+\frac{(\Delta t)^{2}}{\varepsilon}\right)
\end{aligned}
$$

where we have used Proposition 2.1, (1.13), (2.3), and (3.9). Replace $v^{n+1}$ with $H\left(u^{n+1}\right)$ and $v^{\varepsilon, n+1}$ with $H_{\varepsilon}\left(u^{\varepsilon, n+1}\right)$, and use (3.16) to get (3.19). 
We may now conclude that $v^{n} \in L^{\infty}(\Omega)$. Indeed, for every point $t_{*}=m T_{0} / M$, $M \geqslant 1$, it follows from (3.19) and Proposition 2.3, as well as the continuous injection of $L^{2}(\Omega)$ into $F$, that

$$
\begin{array}{ll}
H_{\varepsilon}\left(u^{\varepsilon}\left(\cdot, t_{*}\right)\right) \rightarrow H\left(u\left(\cdot, t_{*}\right)\right) & (\text { in } F), \\
H_{\varepsilon}\left(u^{\varepsilon}\left(\cdot, t_{*}\right)\right) \rightarrow H\left(u\left(\cdot, t_{*}\right)\right) & \text { (weakly in } \left.L^{2}(\Omega)\right),
\end{array}
$$

if $\varepsilon=\Delta t=\left(m T_{0} / j M\right), j \rightarrow \infty$, is selected and $t_{*}=j \Delta t$ is fixed. Since $\left\|H_{\varepsilon}\left(u^{\varepsilon}\left(\cdot, t_{*}\right)\right)\right\|_{L^{\infty}(\Omega)} \leqslant C$ by Proposition 2.3 , this pointwise bound is inherited by the weak $L^{2}$-limit, $H\left(u\left(\cdot, t_{*}\right)\right)$, as is seen by an application of Lusin's theorem to the contrapositive formulation.

The proof of (3.20) requires an alternate bound for term (IV):

$$
\begin{aligned}
& \left|\frac{1}{\Delta t}\left(\int_{t_{n}}^{t_{n+1}}\left(\tau-t_{n}\right)\left[T\left(v^{\varepsilon}-v\right)\right]_{t t}(\tau) d \tau, v^{\varepsilon, n+1}-v^{n+1}\right)_{L^{2}(\Omega)}\right| \\
& \quad \leqslant \frac{1}{\Delta t} \int_{t_{n}}^{t_{n+1}}\left(\tau-t_{n}\right)\left\|\left[T\left(v^{\varepsilon}-v\right)\right]_{t t}(\tau)\right\|_{L^{\prime}(\Omega)} d \tau \cdot\left\|v^{\varepsilon, n+1}-v^{n+1}\right\|_{L^{\infty}(\Omega)} \\
& \quad \leqslant \frac{C}{\Delta t} \cdot(\Delta t)^{2} \cdot\left\|\left[T\left(v^{\varepsilon}-v\right)\right]_{t t}\right\|_{L^{\infty}\left(t_{n}, t_{n+1} ; M(\bar{\Omega})\right)} \\
& \quad \leqslant C(\Delta t)\left\|v_{t}^{\varepsilon}-v_{t}\right\|_{L^{\infty}\left(t_{n}, t_{n+1} ; M(\bar{\Omega})\right)}
\end{aligned}
$$

Using this new bound for (IV), (3.27) becomes

$$
\begin{aligned}
\frac{1}{2 \Delta t}\left\{\| v^{\varepsilon, n+1}-\right. & \left.v^{n+1}\left\|_{F}^{2}-\right\| v^{\varepsilon, n}-v^{n} \|_{F}^{2}\right\} \\
& +\frac{1}{2}\left(J_{\varepsilon}\left(v^{\varepsilon, n+1}\right)-J_{\varepsilon}\left(v^{n+1}\right), v^{\varepsilon, n+1}-v^{n+1}\right)_{L^{2}(\Omega)} \\
\leqslant & C\left[\varepsilon+(\Delta t)\left\|v_{t}^{\varepsilon}-v_{t}\right\|_{L^{\infty}\left(t_{n}, t_{n+1} ; M(\bar{\Omega})\right)}+\left\|v^{\varepsilon, n+1}-v^{n+1}\right\|_{F}^{2}\right] .
\end{aligned}
$$

Modify the second term on the left as before, multiply by $\Delta t$, and sum on $n$ using the discrete Gronwall lemma to obtain

$$
\begin{aligned}
\underset{n}{\operatorname{Max}} & \left\|v^{\varepsilon, n+1}-v^{n+1}\right\|_{F}^{2}+\eta \sum_{n=0}^{M-1}\left(J_{\varepsilon}\left(v^{\varepsilon, n+1}\right)-J\left(v^{n+1}\right), v^{\varepsilon, n+1}-v^{n+1}\right)_{L^{2}(\Omega)} \Delta t \\
& \leqslant C \varepsilon+C(\Delta t)\left\|v_{t}^{\varepsilon}-v_{t}\right\|_{L^{\infty}\left(0, T_{0} ; M(\bar{\Omega})\right)}+C\left\|H_{\varepsilon}\left(u_{0}\right)-H\left(u_{0}\right)\right\|_{F}^{2} \\
& \leqslant C(\varepsilon+\Delta t),
\end{aligned}
$$

where we have used (2.22) and (3.9). Reverting to the notation of (1.1) and (2.1) and using (3.16), we obtain (3.20).

4. Error Estimates for a Continuous Time Galerkin Scheme. Let $\left\{\Delta_{h}\right\}_{h>0}$ be a family of triangulations of $\Omega$ (cf. [9]). For $\tau \in \Delta_{h}$ we define $\rho(\tau)$ (respectively $\sigma(\tau)$ ) to be the radius of the smallest ball containing $\tau$ (respectively largest ball contained in $\tau)$. For convenience we shall assume that

$$
\Omega=\Omega_{h}=\bigcup_{\tau \in \Delta_{h}} \tau
$$


where the boundary elements may have a curvilinear edge coinciding with $\partial \Omega$. Let

$$
M_{h}=\left\{\chi \in C^{0}(\bar{\Omega}):\left.\chi\right|_{\tau} \text { linear, for all } \tau \in \Delta_{h}\right\},
$$

where $h=\sup _{\tau \in \Delta_{h}} \rho(\tau)$. We shall assume that $\left\{M_{h}\right\}_{h>0}$ has the approximation property (cf. [37])

$$
\operatorname{Inf}_{\chi \in M_{h}}\|\zeta-\chi\|_{H^{j}(\Omega)} \leqslant C h^{2-j}\|\zeta\|_{H^{2}(\Omega)} \quad \text { for all } \zeta \in H^{2}(\Omega), j=0,1 .
$$

We shall sometimes need to assume that the underlying triangulations are quasiuniform, i.e., for all $h>0$,

$$
\operatorname{Inf}_{\tau \in \Delta_{h}} \frac{\sigma(\tau)}{h} \geqslant \gamma_{0}>0,
$$

where $\gamma_{0}$ is independent of $h$. The condition (4.2) is known to imply the following inverse hypothesis [9]: there exists a constant $C$, independent of $h$, such that

$$
\|\chi\|_{H^{\prime}(\Omega)} \leqslant \frac{C}{h}\|\chi\|_{L^{2}(\Omega)} \quad \text { for all } \chi \in M_{h} .
$$

We shall restrict ourselves to the use of continuous piecewise-linear elements because the regularity theory presented in Section 2 for the solution of (1.8) is not strong enough to justify the use of spaces with higher order approximation properties. Moreover, the effectiveness of $C^{0}$ piecewise-linear finite element approximation has been demonstrated in [39], [40].

Let $U_{n}:[0, T] \rightarrow M_{h}$ be the solution of the ordinary differential equation

$$
\left(\frac{\partial}{\partial t} H_{\varepsilon}\left(U_{h}\right), \chi\right)_{L^{2}(\Omega)}+\left(\nabla U_{h}, \nabla \chi\right)_{L^{2}(\Omega)}+\left(f\left(U_{h}\right), \chi\right)_{L^{2}(\Omega)}=0,
$$

for all $\chi \in M_{h}, 0<t \leqslant T_{0}$, subject to the initial condition

$$
U_{h}(\cdot, 0)=U_{h}(0)
$$

where $U_{h}(0) \in M_{h}$ is the unique solution of

$$
P_{h} H_{\varepsilon}\left(U_{h}(0)\right)=P_{h} H_{\varepsilon}\left(u^{\varepsilon}(\cdot, 0)\right)=P_{h} H_{\varepsilon}\left(u_{0}\right) .
$$

Here $\varepsilon$ is a nonnegative parameter to be determined later and $P_{h}$ is defined to be the orthogonal projection of $L^{2}(\Omega)$ onto $M_{h}$. The existence and uniqueness of $U_{h}(0)$ when $\varepsilon$ is positive is a consequence of the theory of maximal monotone operators [5]; $P_{h} \circ H_{\varepsilon}$ is a continuous, coercive, monotone operator from $M_{h}$ into itself. The existence of a unique solution of (4.4) for $0<t \leqslant T_{0}$ follows from the fundamental theorem of ordinary differential equations, since $H_{\varepsilon}^{\prime}$ and $f$ are Lipschitz continuous with the former bounded above zero.

Remark 4.1. We shall also require in this section unique solutions of (4.4) when $\varepsilon=0$, i.e., when $H_{\varepsilon}$ is replaced by $H$. As in the case of Eq. (1.8), unique solutions exist in the class $\mathcal{C}$. Solutions of (4.4iii) follow from the theory of (multivalued) maximal monotone operators [5]. In this case, the time derivative of $H\left(U_{h}\right)$ is not necessarily a function. Although the initial function (4.4iii) is convenient for our analysis, it is more practical to choose

$$
U_{h}(0)=E_{h} u_{0}
$$

where $E_{h}$ is the projection of $H^{1}(\Omega)$ onto $M_{h}$ defined in (4.9) below. The additional analysis needed to treat the second choice of initial data will be given below (cf. Remarks 4.5, 4.8-4.11). 
We shall assemble certain facts here which will be used in the derivation of the error estimates later in this section. We begin with a counterpart $T_{h}$ of the operator $T$ which maps $F$ continuously onto $M_{h}$.

Definition 4.1. Given $l \in F$, we define $w_{h}=T_{h} l \in M_{h}$ as the unique element satisfying

$$
\begin{gathered}
\left(\nabla w_{h}, \nabla \chi\right)=\langle l, \chi\rangle-\frac{1}{|\Omega|}\langle l, 1\rangle \int_{\Omega} \chi, \quad \text { all } \chi \in M_{h}, \\
\int_{\Omega} w_{h} d x=\langle l, 1\rangle .
\end{gathered}
$$

Remark 4.2. Combining (4.6i, ii), we see that $w_{h}$ is the Riesz representer, in the inner product (1.10) on $M_{h}$, of the restriction of $l$ to $M_{h}$. This permits us to write

$$
T_{h}=E_{h} T
$$

where

$$
T_{h}: F \rightarrow M_{h},
$$

and $E_{h}$ is the $H^{1}$ projection onto $M_{h}$ defined by

$$
\begin{gathered}
\left(\nabla E_{h} \phi, \nabla \chi\right)_{L^{2}(\Omega)}=(\nabla \phi, \nabla \chi)_{L^{2}(\Omega)}, \quad \text { for all } \chi \in M_{h}, \\
\int_{\Omega} E_{h} \phi d x=\int_{\Omega} \phi d x
\end{gathered}
$$
for $\phi \in H^{1}(\Omega)$. It is immediate from (4.7) that $w_{h}=T_{h} l$ is the standard $H^{1}$ (Galerkin) approximation to $w=T l$, i.e.,

$$
w_{h}=E_{h} w .
$$

In the case where $l=\psi \in L^{2}(\Omega)$, the approximation property

$$
\left\|\left(T-T_{h}\right) \psi\right\|_{L^{2}(\Omega)}=\left\|w-w_{h}\right\|_{L^{2}(\Omega)} \leqslant C h^{2}\|w\|_{H^{2}(\Omega)} \leqslant C h^{2}\|\psi\|_{L^{2}(\Omega)}
$$

is known to hold (cf. [9], [38]), where we have used the relation

$$
\|T \psi\|_{H^{2}(\Omega)} \leqslant C\|\psi\|_{L^{2}(\Omega)} .
$$

Finally, it is possible to obtain a relation analogous to (2.3) by comparing (4.4i) and (4.6i, ii):

$$
\frac{\partial}{\partial t} T_{h} H_{\varepsilon}\left(U_{h}\right)+U_{h}+T_{h} f\left(U_{h}\right)=\frac{1}{|\Omega|} \int_{\Omega} U_{h} d x
$$

a.e. in $\Omega$, for each $0<t \leqslant T_{0}$.

Remark 4.3. The restriction of $T_{h}$ to $L^{2}(\Omega)$ is selfadjoint and nonnegative-definite and the restriction to $M_{h}$ is positive-definite. Thus, we may define an inner product on $M_{h}$ denoted by the subscript $F_{h}$ by

$$
(\psi, \phi)_{F_{h}}=\left(T_{h} \psi, \phi\right)_{L^{2}(\Omega)} \text { for all } \psi, \phi \in M_{h},
$$

from which we obtain the norm

$$
\|\zeta\|_{F_{h}}=\left(T_{h} \zeta, \zeta\right)_{L^{2}(\Omega)}^{1 / 2} \quad \text { for all } \zeta \in M_{h} .
$$

We may extend $\|\cdot\|_{F_{h}}$ to a seminorm on $F$ by defining the associated (semi) inner product,

$$
\left(l_{1}, l_{2}\right)_{F_{h}}=\left\langle l_{1}, T_{h} l_{2}\right\rangle \text { for all } l_{1}, l_{2} \in F
$$


In particular, if $\zeta, \psi \in L^{2}(\Omega)$, then

(4.14) $\quad(\zeta, \psi)_{F_{h}}=\left(T_{h} \zeta, \psi\right)_{L^{2}(\Omega)}=\left(T_{h} \zeta, P_{h} \psi\right)_{L^{2}(\Omega)}=\left(T_{h} P_{h} \zeta, P_{h} \psi\right)_{L^{2}(\Omega)}$.

Our final preliminary result compares $\|\cdot\|_{F_{h}}$ and $\|\cdot\|_{F}$ on $M_{h}$.

Proposition 4.1. The inequality

$$
\|\chi\|_{F_{h}} \leqslant\|\chi\|_{F} \text { for all } \chi \in M_{h},
$$

is valid for $h>0$. If the quasiuniformity hypothesis (4.2) holds, then there is a constant $C$, independent of $h$, such that

$$
\|\chi\|_{F} \leqslant C\|\chi\|_{F_{h}} \text { for all } \chi \in M_{h},
$$

is valid for $h>0$. Thus, the norms are equivalent on $M_{h}$ with constants independent of $h$ in this case.

Proof. We first establish (4.15i).

$$
\begin{aligned}
\|\chi\|_{F_{h}} & =\left(T_{h} \chi, \chi\right)_{L^{2}(\Omega)}^{1 / 2}=\left\|T_{h} \chi\right\|_{H^{1}(\Omega)} \\
& =\left\|E_{h} T \chi\right\|_{H^{1}(\Omega)} \leqslant\|T \chi\|_{H^{1}(\Omega)}=(T \chi, \chi)_{L^{2}(\Omega)}^{1 / 2}=\|\chi\|_{F},
\end{aligned}
$$

where we have used the fact that $\left\|E_{h}\right\| \leqslant 1$ as an operator on $H^{1}(\Omega)$. To establish (4.15ii) we first note that

$$
\|\chi\|_{L^{2}(\Omega)} \leqslant \frac{C}{h}\|\chi\|_{F_{h}} \text { for all } \chi \in M_{h},
$$

where $C$ is the same constant appearing in (4.3). By the latter we have

$$
\begin{aligned}
\|\chi\|_{L^{2}(\Omega)} & =\sup \left\{(\chi, \psi)_{L^{2}(\Omega)}: \psi \in M_{h},\|\psi\|_{L^{2}(\Omega)} \leqslant 1\right\} \\
& \leqslant \sup \left\{\|\chi\|_{F_{h}}\|\psi\|_{H^{1}(\Omega)}: \psi \in M_{h},\|\psi\|_{L^{2}(\Omega)} \leqslant 1\right\} \\
& \leqslant \sup \left\{\|\chi\|_{F_{h}} \frac{C}{h}\|\psi\|_{L^{2}(\Omega)}: \psi \in M_{h},\|\psi\|_{L^{2}(\Omega)} \leqslant 1\right\} \\
& \leqslant \frac{C}{h}\|\chi\|_{F_{h}},
\end{aligned}
$$

which establishes (4.16i). We estimate $\|x\|_{F}$ :

(4.16iii) $\|\chi\|_{F}^{2}=(T \chi, \chi)_{L^{2}(\Omega)}=\left(E_{h} T \chi, \chi\right)_{L^{2}(\Omega)}+\left(\left(I-E_{h}\right) T \chi, \chi\right)_{L^{2}(\Omega)}$

$$
\begin{aligned}
& \leqslant\|\chi\|_{F_{h}}^{2}+\left\|\left(T-T_{h}\right) \chi\right\|_{L^{2}(\Omega)}\|\chi\|_{L^{2}(\Omega)} \\
& \leqslant\|\chi\|_{F_{h}}^{2}+C h^{2}\|\chi\|_{L^{2}(\Omega)}^{2} \leqslant C\|\chi\|_{F_{h}}^{2},
\end{aligned}
$$

where we have used (4.11) and (4.16i).

THEOREM 4.2. Let $u$ be the solution of (1.8) and $U_{h}$ the solution of (4.4). Then for $\varepsilon=\varepsilon_{0} h^{4 / 3}$, any $\varepsilon_{0}>0$, we have

$$
\text { (4.17) } \begin{aligned}
\left\|u-U_{h}\right\|_{L^{2}(D)} \leqslant C & {\left[\int_{0}^{T_{0}}\left(H_{\varepsilon}\left(u^{\varepsilon}\right)-H_{\varepsilon}(u), u^{\varepsilon}-u\right)_{L^{2}(\Omega)} d t\right.} \\
& \left.+\int_{0}^{T_{0}}\left(H_{\varepsilon}\left(u^{\varepsilon}\right)-H_{\varepsilon}\left(U_{h}\right), u^{\varepsilon}-U_{h}\right)_{L^{2}(\Omega)} d t\right]^{1 / 2} \\
\leqslant & C h^{2 / 3},
\end{aligned}
$$


for some positive constant $C$. If we assume the quasiuniformity hypothesis (4.2), then

$$
\left\|H(u)-P_{h} H_{\varepsilon}\left(U_{h}\right)\right\|_{L^{\infty}(0, T ; F)} \leqslant C h^{2 / 3},
$$

where the $F$ norm was defined in (1.12).

Proof. Subtraction of (4.12) from (2.3) yields

$$
\begin{aligned}
\frac{\partial}{\partial t} T_{h}[ & \left.H_{\varepsilon}\left(u^{\varepsilon}\right)-H_{\varepsilon}\left(U_{h}\right)\right]+\left(u^{\varepsilon}-U_{h}\right) \\
= & -T_{h}\left[f\left(u^{\varepsilon}\right)-f\left(U_{h}\right)\right]+\left(T_{h}-T\right)\left[\frac{\partial}{\partial t} H_{\varepsilon}\left(u^{\varepsilon}\right)+f\left(u^{\varepsilon}\right)\right] \\
& +\frac{1}{|\Omega|} \int_{\Omega}\left(u^{\varepsilon}-U_{h}\right)(x, t) d x .
\end{aligned}
$$

Integrate this against $H_{\varepsilon}\left(u^{\varepsilon}\right)-H_{\varepsilon}\left(U_{h}\right)$ over $\Omega$ to obtain

$$
\begin{aligned}
\frac{1}{2} \frac{d}{d t}\left\|H_{\varepsilon}\left(u^{\varepsilon}\right)-H_{\varepsilon}\left(U_{h}\right)\right\|_{F_{h}}^{2}+\left(H_{\varepsilon}\left(u^{\varepsilon}\right)-H_{\varepsilon}\left(U_{h}\right), u^{\varepsilon}-U_{h}\right)_{L^{2}(\Omega)} \\
=-\left(T_{h}\left[f\left(u^{\varepsilon}\right)-f\left(U_{h}\right)\right], H_{\varepsilon}\left(u^{\varepsilon}\right)-H_{\varepsilon}\left(U_{h}\right)\right)_{L^{2}(\Omega)} \\
+\left(\left(T_{h}-T\right)\left[\frac{\partial}{\partial t} H_{\varepsilon}\left(u^{\varepsilon}\right)+f\left(u^{\varepsilon}\right)\right], H_{\varepsilon}\left(u^{\varepsilon}\right)-H_{\varepsilon}\left(U_{h}\right)\right)_{L^{2}(\Omega)} \\
\quad+\frac{1}{|\Omega|} \int_{\Omega}\left(u^{\varepsilon}-U_{h}\right) d x \int_{\Omega}\left(H_{\varepsilon}\left(u^{\varepsilon}\right)-H_{\varepsilon}\left(U_{h}\right)\right) d x \\
=(\mathrm{I})+(\mathrm{II})+(\mathrm{III}) .
\end{aligned}
$$

We bound the first term on the right-hand side of (4.19) as follows.

$$
\begin{gathered}
\left|\left(f\left(u^{\varepsilon}\right)-f\left(U_{h}\right), H_{\varepsilon}\left(u^{\varepsilon}\right)-H_{\varepsilon}\left(U_{h}\right)\right)_{F_{h}}\right| \leqslant\left\|f\left(u^{\varepsilon}\right)-f\left(U_{h}\right)\right\|_{F_{h}}\left\|H_{\varepsilon}\left(u^{\varepsilon}\right)-H_{\varepsilon}\left(U_{h}\right)\right\|_{F_{h}} \\
\leqslant C\left\|u^{\varepsilon}-U_{h}\right\|_{L^{2}(\Omega)}\left\|H_{\varepsilon}\left(u^{\varepsilon}\right)-H_{\varepsilon}\left(U_{h}\right)\right\|_{F_{h}}
\end{gathered}
$$

by the Lipschitz continuity of $f,(4.15 i)$, and the continuity of the injection of $L^{2}(\Omega)$ into $F$. Thus, for some $C$,

$$
|\mathrm{I}| \leqslant \frac{1}{2} \eta \lambda^{-1}\left(H_{\varepsilon}\left(u^{\varepsilon}\right)-H_{\varepsilon}\left(U_{h}\right), u^{\varepsilon}-U_{h}\right)_{L^{2}(\Omega)}+C \eta^{-1}\left\|H_{\varepsilon}\left(u^{\varepsilon}\right)-H_{\varepsilon}\left(U_{h}\right)\right\|_{F_{h}}^{2},
$$

where we have used (3.3). For $\eta$ sufficiently small, say $\eta=\lambda / 3$, the first term on the right-hand side of (4.20) can be absorbed in the second term on the left-hand side of (4.19).

Prior to estimating (II) we note the inequality

$$
\left(H_{\varepsilon}(\zeta)-H_{\varepsilon}(\psi), \zeta-\psi\right)_{L^{2}(\Omega)} \geqslant(\varepsilon / \gamma)\left\|H_{\varepsilon}(\zeta)-H_{\varepsilon}(\psi)\right\|_{L^{2}(\Omega)}^{2}
$$

for $\zeta$ and $\psi$ in $L^{2}(\Omega)$, which follows directly from (1.18). We use (4.11) and inequality (4.21) to bound (II).

$$
\begin{aligned}
& |(\mathrm{II})| \leqslant\left\|\left(T-T_{h}\right)\left[\frac{\partial}{\partial t} H_{\varepsilon}\left(u^{\varepsilon}\right)+f\left(u^{\varepsilon}\right)\right]\right\|_{L^{2}(\Omega)}\left\|H_{\varepsilon}\left(u^{\varepsilon}\right)-H_{\varepsilon}\left(U_{h}\right)\right\|_{L^{2}(\Omega)} \\
& \leqslant \eta^{-1} \varepsilon^{-1} h^{4}\left[\left\|\frac{\partial}{\partial t} H_{\varepsilon}\left(u^{\varepsilon}\right)\right\|_{L^{2}(\Omega)}^{2}+C\left\|u^{\varepsilon}\right\|_{L^{2}(\Omega)}^{2}\right]+C \eta \varepsilon\left\|H_{\varepsilon}\left(u^{\varepsilon}\right)-H_{\varepsilon}\left(U_{h}\right)\right\|_{L^{2}(\Omega)}^{2} .
\end{aligned}
$$


Note that for a given $\varepsilon$, the choice $\eta=1 /(6 C \gamma)$, together with (4.21), permits the last term to be absorbed in the left side of (4.19). Since $T_{h}$ preserves mean values, the Cauchy-Schwarz inequality yields

$$
|(\mathrm{III})| \leqslant C\left\|u^{\varepsilon}-U_{h}\right\|_{L^{2}(\Omega)}\left\|H_{\varepsilon}\left(u^{\varepsilon}\right)-H_{\varepsilon}\left(U_{h}\right)\right\|_{F_{h}},
$$

so that, for some constant $C$, (III) is bounded by

$$
\begin{aligned}
|(\mathrm{III})| \leqslant & \frac{1}{2} \eta \lambda^{-1}\left(H_{\varepsilon}\left(u^{\varepsilon}\right)-H_{\varepsilon}\left(U_{h}\right), u^{\varepsilon}-U_{h}\right)_{L^{2}(\Omega)} \\
& +\eta^{-1} C\left\|H_{\varepsilon}\left(u^{\varepsilon}\right)-H_{\varepsilon}\left(U_{h}\right)\right\|_{F_{h}}^{2},
\end{aligned}
$$

and the choice $\eta=\lambda / 3$ permits the first term on the right-hand side of (4.23) to be absorbed on the left-hand side of (4.19). Applying (4.20), (4.22), and (4.23) to (4.19), we have

$$
\begin{aligned}
& \frac{1}{2} \frac{d}{d t}\left\|H_{\varepsilon}\left(u^{\varepsilon}\right)-H_{\varepsilon}\left(U_{h}\right)\right\|_{F_{h}}^{2}+\frac{1}{2}\left(H_{\varepsilon}\left(u^{\varepsilon}\right)-H_{\varepsilon}\left(U_{h}\right), u^{\varepsilon}-U_{h}\right)_{L^{2}(\Omega)} \\
& \quad \leqslant C \frac{h^{4}}{\varepsilon}\left[\left\|\frac{\partial}{\partial t} H_{\varepsilon}\left(u^{\varepsilon}\right)\right\|_{L^{2}(\Omega)}^{2}+\left\|u^{\varepsilon}\right\|_{L^{2}(\Omega)}^{2}\right]+C\left\|H_{\varepsilon}\left(u^{\varepsilon}\right)-H_{\varepsilon}\left(U_{h}\right)\right\|_{F_{h}}^{2} .
\end{aligned}
$$

Integrating (4.24) in time and using the Gronwall inequality, we obtain

$$
\begin{gathered}
\left\|H_{\varepsilon}\left(u^{\varepsilon}\right)-H_{\varepsilon}\left(U_{h}\right)\right\|_{L^{\infty}\left(0, T_{0} ; F_{h}\right)}^{2}+\int_{0}^{T_{0}}\left(H_{\varepsilon}\left(u^{\varepsilon}\right)-H_{\varepsilon}\left(U_{h}\right), u^{\varepsilon}-U_{h}\right)_{L^{2}(\Omega)} d t \\
\leqslant C \frac{h^{4}}{\varepsilon}\left[\left\|\frac{\partial}{\partial t} H_{\varepsilon}\left(u^{\varepsilon}\right)\right\|_{L^{2}(D)}^{2}+\left\|u^{\varepsilon}\right\|_{L^{2}(\Omega)}^{2}\right] .
\end{gathered}
$$

Notice that the $F_{h}$ seminorm of $H_{\varepsilon}\left(u^{\varepsilon}(\cdot, 0)\right)-H_{\varepsilon}\left(U_{h}(0)\right)$ is 0 since the projection of this function onto $M_{h}$ is 0 by (4.4iii). Now, by (2.4iii), we obtain from (4.25) the estimate

$$
\begin{aligned}
\| H_{\varepsilon}\left(u^{\varepsilon}\right)- & H_{\varepsilon}\left(U_{h}\right) \|_{L^{\infty}\left(0, T_{0} ; F_{h}\right)}^{2} \\
& +\int_{0}^{T_{0}}\left(H_{\varepsilon}\left(u^{\varepsilon}\right)-H_{\varepsilon}\left(U_{h}\right), u^{\varepsilon}-U_{h}\right)_{L^{2}(\Omega)} d t \leqslant C \frac{h^{4}}{\varepsilon^{2}} .
\end{aligned}
$$

Comparing (4.26) with the estimate (3.10ii), we have, by (3.3),

$$
\begin{aligned}
\left\|u-U_{h}\right\|_{L^{2}(D)}^{2} \leqslant & C \int_{0}^{T_{0}}\left(u^{\varepsilon}-u, u^{\varepsilon}-u\right)_{L^{2}(\Omega)} d t \\
& +C \int_{0}^{T_{0}}\left(H_{\varepsilon}\left(u^{\varepsilon}\right)-H_{\varepsilon}\left(U_{h}\right), u^{\varepsilon}-U_{h}\right)_{L^{2}(\Omega)} d t \\
\leqslant & C\left(\varepsilon+\frac{h^{4}}{\varepsilon^{2}}\right) \leqslant C h^{4 / 3},
\end{aligned}
$$

if the choice $\varepsilon=\varepsilon_{0} h^{4 / 3}$ is made for some positive constant $\varepsilon_{0}$. This yields (4.17).

For the remainder of the proof we assume the quasiuniformity hypothesis (4.2) and hence the inverse hypothesis (4.3) and inequality (4.15ii). Using the latter inequality, we may rewrite (4.26) as

$$
\begin{aligned}
\delta^{2} \| P_{h}[ & \left.H_{\varepsilon}\left(u^{\varepsilon}\right)-H_{\varepsilon}\left(U_{h}\right)\right] \|_{L^{\infty}\left(0, T_{0} ; F\right)}^{2} \\
& +\int_{0}^{T_{0}}\left(H_{\varepsilon}\left(u^{\varepsilon}\right)-H_{\varepsilon}\left(U_{h}\right), u^{\varepsilon}-U_{h}\right)_{L^{2}(\Omega)} d t \leqslant C \frac{h^{4}}{\varepsilon^{2}} .
\end{aligned}
$$


It remains to estimate $\left(I-P_{h}\right)\left(H_{\varepsilon}\left(u^{\varepsilon}\right)-H_{\varepsilon}\left(U_{h}\right)\right)$ in the $L^{\infty}\left(0, T_{0} ; F\right)$ norm. However, this term is of order $O(h)$ (cf. (4.30) to follow) so that (4.18) follows upon combining this estimate with (4.27) with the choice $\varepsilon=\varepsilon_{0} h^{4 / 3}$.

Remark 4.4. The quasiuniformity hypothesis in Theorem 4.2 and Corollary 4.3 can be removed. This follows from the inequality

$$
\begin{aligned}
\|f\|_{F}^{2} & =(T f, f)_{L^{2}(\Omega)}=\left(T_{h} f, f\right)_{L^{2}(\Omega)}+\left(\left(T-T_{h}\right) f, f\right)_{L^{2}(\Omega)} \\
& \leqslant\|f\|_{F_{h}}^{2}+\left\|\left(T-T_{h}\right) f\right\|_{L^{2}(\Omega)}\|f\|_{L^{2}(\Omega)} \\
& \leqslant\|f\|_{F_{h}}^{2}+C h^{2}\|f\|_{L^{2}(\Omega)}^{2},
\end{aligned}
$$

for $f \in L^{2}(\Omega)$, applied to $f=H_{\varepsilon}\left(u^{\varepsilon}\right)-H_{\varepsilon}\left(U_{h}\right)$. Proposition 2.3 and estimate (4.32), to follow, give $L^{\infty}\left(\left(0, T_{0}\right) ; L^{2}(\Omega)\right)$ bounds for the latter.

COROLlaRY 4.3. Under the hypotheses of Theorem 4.2, including the quasiuniformity hypothesis (4.2), the estimate

$$
\left\|H(u)-H_{\varepsilon}\left(U_{h}\right)\right\|_{L^{\infty}\left(0, T_{0} ; F\right)} \leqslant C h^{2 / 3}
$$

holds if the choice $\varepsilon=\varepsilon_{0} h^{4 / 3}$ is made, $\varepsilon_{0}>0$, for some $C>0$.

Proof. Given (3.10i) and (4.27), it suffices to establish the estimate

$$
\left\|\left(I-P_{h}\right)\left[H_{\varepsilon}\left(u^{\varepsilon}\right)-H_{\varepsilon}\left(U_{h}\right)\right]\right\|_{L^{\infty}\left(0, T_{0} ; F\right)} \leqslant C h
$$

for some $C>0$ independent of $h$. However, a standard duality result gives

$$
\begin{aligned}
& \left\|\left(I-P_{h}\right)\left[H_{\varepsilon}\left(u^{\varepsilon}\right)-H_{\varepsilon}\left(U_{h}\right)\right]\right\|_{L^{\infty}\left(0, T_{0} ; F\right)} \\
& \quad \leqslant C h\left\|H_{\varepsilon}\left(u^{\varepsilon}\right)-H_{\varepsilon}\left(U_{h}\right)\right\|_{L^{\infty}\left(0, T_{0} ; L^{2}(\Omega)\right)} .
\end{aligned}
$$

Now, given (2.4i), it suffices to establish the estimate

$$
\left\|H_{\varepsilon}\left(U_{h}\right)\right\|_{L^{\infty}\left(0, T_{0} ; L^{2}(\Omega)\right)} \leqslant A|\Omega|+\mu\left\|U_{h}\right\|_{L^{\infty}\left(0, T_{0} ; L^{2}(\Omega)\right)} \leqslant C .
$$

However, (4.32) is derived from (4.4) by setting $\chi=U_{h}$ and integrating in time. Specifically, with this substitution, the first term of (4.4i) assumes the form

$$
\left(\frac{\partial H_{\varepsilon}\left(U_{h}\right)}{\partial t}, U_{h}\right)_{L^{2}(\Omega)}=\frac{d}{d t} \int_{\Omega}\left\{\int_{0}^{U_{h}} H_{\varepsilon}^{\prime}(\xi) \xi d \xi\right\},
$$

and integration in time yields

$$
\begin{aligned}
\int_{0}^{t}\left(\frac{\partial H_{\varepsilon}\left(U_{h}\right)}{\partial t}, U_{h}\right)_{L^{2}(\Omega)} d \tau & =\int_{\Omega}\left\{\int_{0}^{U_{h}(t)} H_{\varepsilon}^{\prime}(\xi) \xi d \xi-\int_{0}^{U_{h}(0)} H_{\varepsilon}^{\prime}(\xi) \xi d \xi\right\} \\
\geqslant & \frac{1}{2} \lambda\left\|U_{h}(t)\right\|_{L^{2}(\Omega)}^{2}-\left\|U_{h}(0)\right\|_{L^{2}(\Omega)}\left\|\int_{0}^{U_{h}(0)} H_{\varepsilon}^{\prime}(\xi) d \xi\right\|_{L^{2}(\Omega)} \\
\geqslant & \frac{1}{2} \lambda\left\|U_{h}(t)\right\|_{L^{2}(\Omega)}^{2}-\left\|U_{h}(0)\right\|_{L^{2}(\Omega)}\left(A|\Omega|+\mu\left\|U_{h}(0)\right\|_{L^{2}(\Omega)}\right) .
\end{aligned}
$$

If the nonnegative second term of (4.4i) and the third term are estimated in the standard fashion, the resultant expression yields, via the Gronwall inequality,

$$
\left\|\nabla U_{h}\right\|_{L^{2}\left(0, T_{0} ; L^{2}(\Omega)\right)}^{2}+\left\|U_{h}\right\|_{L^{\infty}\left(0, T_{0} ; L^{2}(\Omega)\right)}^{2} \leqslant C_{1}+C_{2}\left\|U_{h}(0)\right\|_{L^{2}(\Omega)}^{2} \leqslant C,
$$

since $\left\{U_{h}(0)\right\}$ is bounded in $L^{2}(\Omega)$ via (4.4iii). Inequality (4.32) is now established. Estimate (4.30) is now immediate from (4.31). 
By setting $\chi=\left(U_{h}\right)_{t}$ in (4.4i) we may obtain a uniform bound for $U_{h}$ in $L^{\infty}\left(0, T_{0} ; H^{1}(\Omega)\right)$ when $U_{h}(0)=E_{h} u_{0}$ (cf. Remark 4.9 and (4.46i)).

Remark 4.5. If the initial function (4.5) is used instead of (4.4iii), then the additional term

$$
\left\|H_{\varepsilon}\left(u_{0}\right)-H_{\varepsilon}\left(U_{h}(0)\right)\right\|_{F_{h}}^{2}
$$

appears on the right side of (4.26). We can show that $(*)$ is $O\left(h^{4} / \varepsilon^{2}\right)$ if we assume that $u_{0} \in H^{2}(\Omega)$, where $u_{0}$ is the initial function in the Stefan problem (1.1). By (1.18), (4.11), and (4.15i),

$$
\begin{aligned}
\left\|H_{\varepsilon}\left(u_{0}\right)-H_{\varepsilon}\left(U_{h}(0)\right)\right\|_{F_{h}} & \leqslant C\left\|H_{\varepsilon}\left(u_{0}\right)-H_{\varepsilon}\left(E_{h} u_{0}\right)\right\|_{L^{2}(\Omega)} \\
& \leqslant \frac{C}{\varepsilon}\left\|\left(I-E_{h}\right) u_{0}\right\|_{L^{2}(\Omega)} \leqslant C \frac{h^{2}}{\varepsilon}\left\|u_{0}\right\|_{H^{2}(\Omega)} \leqslant C \frac{h^{2}}{\varepsilon} .
\end{aligned}
$$

An alternative hypothesis is given by the following analogue of (3.9): for $\varepsilon \leqslant \varepsilon_{0} h^{2}$, some $\varepsilon_{0}>0$,

$$
\mid\left\{x \in \Omega: 0<u_{0}(x) \leqslant \varepsilon \text { or } 0<\left(E_{h} u_{0}\right)(x) \leqslant \varepsilon\right\}|=| A_{\varepsilon, h} \mid \leqslant C \varepsilon .
$$

This implies, for positive $\varepsilon$ sufficiently small,

$$
\begin{aligned}
& \left\|H_{\varepsilon}\left(u_{0}\right)-H_{\varepsilon}\left(E_{h} u_{0}\right)\right\|_{L^{2}(\Omega)}^{2} \\
& \quad=\left\|H_{\varepsilon}\left(u_{0}\right)-H_{\varepsilon}\left(E_{h} u_{0}\right)\right\|_{L^{2}\left(A_{\varepsilon, h}\right)}^{2}+\left\|H_{\varepsilon}\left(u_{0}\right)-H_{\varepsilon}\left(E_{h} u_{0}\right)\right\|_{L^{2}\left(\Omega \sim A_{\varepsilon, h}\right)}^{2} \\
& \quad \leqslant C \varepsilon+C\left\|\left(I-E_{h}\right) u_{0}\right\|_{L^{2}(\Omega)}^{2} \leqslant C\left(\varepsilon+h^{2}\right) \leqslant C h^{2} .
\end{aligned}
$$

We will use the fact that $(* *)$ implies

$$
\left\|H_{\varepsilon}\left(u_{0}\right)-H_{\varepsilon}\left(E_{h} u_{0}\right)\right\|_{F_{h}} \leqslant C h
$$

to verify the conclusions of Theorems 4.5 and 4.6 when the initial function is given by (4.5). The case $H_{0}=H$ requires a modified form of (**) at 0 (cf. (3.9)).

Remark 4.6. Although one must choose $\varepsilon$ to be proportional to $h^{4 / 3}$ to obtain $O\left(h^{2 / 3}\right)$ convergence in the proof of Theorem 4.2, under a different choice of $\varepsilon$ one can achieve first-order convergence of the scheme (4.4). Under a very plausible assumption on the $L^{\infty}$ boundedness of $H_{\varepsilon}\left(U_{h}\right)$, we shall show that the scheme (4.4) is essentially $O(h)$-accurate with any $\varepsilon$ satisfying $0 \leqslant \varepsilon \leqslant \varepsilon_{0} h^{2}$ for some positive constant $\varepsilon_{0}$. Because $\partial H(u) / \partial t$ is known only to be a finite regular Baire measure of bounded total variation, it will be necessary to estimate (II) in the proof of the previous theorem by $L^{\infty}(\Omega)-M(\bar{\Omega})$ duality. By $M(\bar{\Omega})$ we mean the finite regular Baire measures, normed with the total variation norm. Although $M(\bar{\Omega})$ is the natural dual of $C(\bar{\Omega})$, the use of the norm-preserving Lebesgue extension [14] permits the inequality

$$
\left|\int_{\bar{\Omega}} \zeta \mu(d s)\right| \leqslant\|\zeta\|_{L^{\infty}(\Omega)}\|\mu\|_{M(\bar{\Omega})} \quad \text { for all } \zeta \in L^{\infty}(\Omega) .
$$

We now cite the required finite element estimates. 
Proposition 4.4. Let $\zeta \in L^{\infty}(\Omega)$. Then there is a constant $C$ such that

(i) $\left\|\left(T-T_{h}\right)\right\|_{L^{\infty}(\Omega)} \leqslant C h^{2}\|\zeta\|_{L^{\infty}(\Omega)}, \quad \operatorname{dim}(\Omega)=1$,

(ii) $\left\|\left(T-T_{h}\right) \zeta\right\|_{L^{\infty}(\Omega)} \leqslant C\left[\ln \left(\frac{1}{h}\right)\right]^{2} h^{2}\|\zeta\|_{L^{\infty}(\Omega)}, \quad \operatorname{dim}(\Omega) \geqslant 2$.

In particular, if $\mu \in M(\bar{\Omega})$, then

$$
\left\|\left(T-T_{h}\right) \mu\right\|_{M(\bar{\Omega})} \leqslant C\left\{\begin{array}{c}
h^{2} \\
h^{2}[\ln (1 / h)]^{2}
\end{array}\right\}\|\mu\|_{M(\bar{\Omega}),}, \quad \begin{array}{ll}
\operatorname{dim}(\Omega)=1, & \operatorname{dim}(\Omega) \geqslant 2 .
\end{array}
$$

Proof. The estimate (4.35i) is due to Douglas, Dupont, and Wahlbin [13] whereas (4.35ii) in exactly the form as stated is due to Schatz and Wahlbin [33], who cite V. Thomée's helpful suggestion. The argument proceeds in stages, the first stage being the estimate, for $h \leqslant 1$,

$$
\left\|\left(T-T_{h}\right) \zeta\right\|_{L^{\infty}(\Omega)} \leqslant C \ln \left(\frac{1}{h}\right) \min _{\chi \in M_{h}}\|T \zeta-\chi\|_{L^{\infty}(\Omega)}
$$

due to R. Scott [34] for the Neumann problem. This is followed by the approximation estimate

$$
\min _{\chi \in M_{h}}\|T \zeta-\chi\|_{L^{\infty}(\Omega)} \leqslant C_{1} h^{2-n / p}\|T \zeta\|_{W^{2, p}(\Omega)} \quad\left(C_{1} \text { ind. of } p>1\right),
$$

which in turn is followed by the Agmon-Douglis-Nirenberg estimate (cf. [1] and [19])

$$
\|T \zeta\|_{W^{2, p}(\Omega)} \leqslant C_{2} p\|f\|_{L^{p}(\Omega)} \quad\left(C_{2} \text { ind. of } p\right) .
$$

Combining these estimates gives

$$
\left\|\left(T-T_{h}\right) \zeta\right\|_{L^{\infty}(\Omega)} \leqslant C_{2} \ln (1 / h)\left(p h^{-n / p}\right) h^{2}|\Omega|^{1 / p}\|f\|_{L^{\infty}(\Omega)},
$$

and (4.35ii) follows if $p h^{-n / p}$ is minimized as a function of $p>1$. A weaker form of (4.35ii) was obtained by Rannacher [28]. Variants of (4.35ii) in which $\|\zeta\|_{L^{\infty}(\Omega)}$ is replaced by more complicated second-order expressions involving $T \zeta$ have been proved by Nitsche [27] and Scott [34]. The estimate (4.36) follows from a simple duality argument:

$$
\begin{aligned}
\left\|\left(T-T_{h}\right) \mu\right\|_{M(\bar{\Omega})} & =\sup \left\{\left|\left\langle\left(T-T_{h}\right) \mu, \psi\right\rangle\right|: \psi \in C(\bar{\Omega}),\|\psi\|_{C(\bar{\Omega})} \leqslant 1\right\} \\
& =\sup \left\{\left|\left\langle\left(T-T_{h}\right) \psi, \mu\right\rangle\right|: \psi \in C(\bar{\Omega}),\|\psi\|_{C(\bar{\Omega})} \leqslant 1\right\},
\end{aligned}
$$

and (4.36) follows directly from applying (4.34) and (4.35) to (4.37).

TheOREM 4.5. Suppose $0 \leqslant \varepsilon \leqslant \varepsilon_{0} h^{2}$. Let $u$ and $U_{h}$ be the solutions of (1.8) and (4.4), respectively. Assume that the quasiuniformity hypothesis (4.2) holds and that

$$
\left\|H_{\varepsilon}\left(U_{h}\right)\right\|_{L^{\infty}(\Omega)} \leqslant C_{0} \text { for all } 0<t \leqslant T_{0},
$$

where $C_{0}$ is independent of $\varepsilon$ and $h$. Then we have the estimates, for $h \leqslant 1$,

$$
\left\|H(u)-H_{\varepsilon}\left(U_{h}\right)\right\|_{L^{\infty}\left(0, T_{0} ; F\right)} \leqslant C \begin{cases}h, & \operatorname{dim}(\Omega)=1, \\ h[\ln (1 / h)], & \operatorname{dim}(\Omega) \geqslant 2,\end{cases}
$$

and

$$
\left\|u-U_{h}\right\|_{L^{2}(D)} \leqslant C \begin{cases}h, & \operatorname{dim}(\Omega)=1, \\ h[\ln (1 / h)], & \operatorname{dim}(\Omega) \geqslant 2 .\end{cases}
$$


Proof. By (4.19), (4.20), (4.22), and (4.23) we obtain

$$
\begin{aligned}
& \frac{1}{2} \frac{d}{d t}\left\|H_{\varepsilon}\left(u^{\varepsilon}\right)-H_{\varepsilon}\left(U_{h}\right)\right\|_{F_{h}}^{2}+\frac{1}{2}\left(H_{\varepsilon}\left(u^{\varepsilon}\right)-H_{\varepsilon}\left(U_{h}\right), u^{\varepsilon}-U_{h}\right)_{L^{2}(\Omega)} \\
& \leqslant\left(\left(T-T_{h}\right) \frac{\partial}{\partial t} H_{\varepsilon}\left(u^{\varepsilon}\right), H_{\varepsilon}\left(u^{\varepsilon}\right)-H_{\varepsilon}\left(U_{h}\right)\right)_{L^{2}(\Omega)} \\
&+C\left\|H_{\varepsilon}\left(u^{\varepsilon}\right)-H_{\varepsilon}\left(U_{h}\right)\right\|_{F_{h}}^{2}+C\left\|\left(T-T_{h}\right) f\left(u^{\varepsilon}\right)\right\|_{L^{2}(\Omega)}^{2} \\
& \leqslant\left\|\left(T-T_{h}\right) \frac{\partial}{\partial t} H_{\varepsilon}\left(u^{\varepsilon}\right)\right\|_{M(\bar{\Omega})}\left\|H_{\varepsilon}\left(u^{\varepsilon}\right)-H_{\varepsilon}\left(U_{h}\right)\right\|_{L^{\infty}(\Omega)} \\
&+C\left\|H_{\varepsilon}\left(u^{\varepsilon}\right)-H_{\varepsilon}\left(U_{h}\right)\right\|_{F_{h}}^{2}+C h^{2} .
\end{aligned}
$$

Integrating in time and using Gronwall's inequality, we obtain

$$
\begin{aligned}
\text { (4.41) } & \left\|H_{\varepsilon}\left(u^{\varepsilon}\right)-H_{\varepsilon}\left(U_{h}\right)\right\|_{L^{\infty}\left(0, T_{0} ; F_{h}\right)}^{2}+\int_{0}^{T_{0}}\left(H_{\varepsilon}\left(u^{\varepsilon}\right)-H_{\varepsilon}\left(U_{h}\right), u^{\varepsilon}-U_{h}\right)_{L^{2}(\Omega)} d t \\
& \leqslant C\left[\left\|\left(T_{h}-T\right) \frac{\partial}{\partial t} H_{\varepsilon}\left(u^{\varepsilon}\right)\right\|_{L^{\infty}\left(0, T_{0} ; M(\bar{\Omega})\right)}\left\|H_{\varepsilon}\left(u^{\varepsilon}\right)-H_{\varepsilon}\left(U_{h}\right)\right\|_{L^{\prime}\left(0, T_{0} ; L^{\infty}(\Omega)\right)}+h^{2}\right] .
\end{aligned}
$$

Using Proposition 2.3 and the assumption (4.38), we conclude that

$$
\left\|H_{\varepsilon}\left(u^{\varepsilon}\right)-H_{\varepsilon}\left(U_{h}\right)\right\|_{L^{1}\left(0, T_{0} ; L^{\infty}(\Omega)\right)} \leqslant C .
$$

Using (4.41) in combination with (4.15ii), (4.36), (4.42), and (2.6) yields

$$
\begin{aligned}
\delta^{2} \| P_{h}\left[H_{\varepsilon}\left(u^{\varepsilon}\right)-\right. & \left.H_{\varepsilon}\left(U_{h}\right)\right] \|_{L^{\infty}\left(0, T_{0} ; F\right)}^{2}+\int_{0}^{T_{0}}\left(H_{\varepsilon}\left(u^{\varepsilon}\right)-H_{\varepsilon}\left(U_{h}\right), u^{\varepsilon}-U_{h}\right)_{L^{2}(\Omega)} d t \\
& \leqslant C \begin{cases}h^{2}, & \operatorname{dim}(\Omega)=1, \\
h^{2}[\ln (1 / h)]^{2}, & \operatorname{dim}(\Omega) \geqslant 2 .\end{cases}
\end{aligned}
$$

Applying (1.18), (3.10), and (4.32) to (4.43), we obtain

$$
\begin{aligned}
\left\|H(u)-H_{\varepsilon}\left(U_{h}\right)\right\|_{L^{\infty}\left(0, T_{0} ; F\right)}^{2}+\left\|u-U_{h}\right\|_{L^{2}(D)}^{2} & \\
& \leqslant C \begin{cases}\left(\varepsilon+h^{2}\right), & \operatorname{dim}(\Omega)=1, \\
\varepsilon+h^{2}[\ln (1 / h)]^{2}, & \operatorname{dim}(\Omega) \geqslant 2 .\end{cases}
\end{aligned}
$$

This yields (4.39) and (4.40).

Remark 4.7. Theorem 4.5 indicates that one may set $\varepsilon=0$ in (4.4) and still obtain essentially first-order convergence. This raises the question of why we introduced the $\varepsilon$-regularization. It turns out that when one introduces the backward-difference time discretization of (4.4), the resulting nonlinear algebraic problem is easier to solve by standard methods with $\varepsilon=\varepsilon_{0} h^{4 / 3}$ than $\varepsilon=0$. Also, the restriction on the size of the time step is less severe.

Remark 4.8. If we assume (**) as in Remark 4.5, then Theorem 4.5 is valid for the initial function (4.5). An obvious modification is required when $\varepsilon=0$.

In some media it is possible that the unfrozen moisture content is a continuously differentiable function of temperature even at $0^{\circ} \mathrm{C}$. In this case the enthalpy function is a continuously differentiable function which satisfies (1.18) for some positive parameter $\varepsilon$; we denote this enthalpy as $H_{\varepsilon}(\zeta)$, where the $\varepsilon$ is now of physical significance. 
If $\varepsilon$ is sufficiently large with respect to the mesh spacing $h$, then estimate (4.26) becomes significant with $u^{\varepsilon}$ representing the physical transformed temperature variable. The same comment applies to the fully discrete estimate (5.14).

Remark 4.9. By (2.4ii), (3.10i), and (3.16),

$$
\begin{aligned}
\lambda\left\|u^{\varepsilon}-u\right\|_{L^{2}(\Omega)}^{2} & \leqslant\left(H_{\varepsilon}\left(u^{\varepsilon}\right)-H(u), u^{\varepsilon}-u\right)_{L^{2}(\Omega)}+C \varepsilon \\
& \leqslant\left\|H_{\varepsilon}\left(u^{\varepsilon}\right)-H(u)\right\|_{L^{\infty}(0, T ; F)} \cdot\left\|u^{\varepsilon}-u\right\|_{L^{\infty}\left(0, T ; H^{1}(\Omega)\right)}+C \varepsilon \\
& \leqslant C \sqrt{\varepsilon}+C \varepsilon \leqslant C \sqrt{\varepsilon}
\end{aligned}
$$

so that

$$
\left\|u^{\varepsilon}-u\right\|_{L^{\infty}\left(0, T_{0} ; L^{2}(\Omega)\right)} \leqslant C \varepsilon^{1 / 4} .
$$

When $U_{h}(0)=E_{h} u_{0}$ and $(* *)$ holds,

$$
\begin{aligned}
& \lambda\left\|u-U_{h}\right\|_{L^{\infty}\left(0, T_{0} ; L^{2}(\Omega)\right)}^{2} \leqslant 2\left(H_{\varepsilon}\left(u^{\varepsilon}\right)-H_{\varepsilon}\left(U_{h}\right), u^{\varepsilon}-U_{h}\right)+C \sqrt{\varepsilon} \\
& \quad \leqslant 2\left\|H_{\varepsilon}\left(u^{\varepsilon}\right)-H_{\varepsilon}\left(U_{h}\right)\right\|_{L^{\infty}\left(0, T_{0} ; F\right)}\left\|u^{\varepsilon}-U_{h}\right\|_{L^{\infty}\left(0, T_{0} ; H^{1}(\Omega)\right)}+C \sqrt{\varepsilon} \\
& \quad \leqslant C\left(\frac{h^{2}}{\varepsilon}+\sqrt{\varepsilon}\right)=C h^{2 / 3}
\end{aligned}
$$

with $\varepsilon=\varepsilon_{0} h^{4 / 3}$, where we have used (1.18), (2.4ii), (2.23ii), (4.26), (4.45), and the bound

$$
\left\|U_{h}\right\|_{L^{\infty}\left(0, T_{0} ; H^{1}(\Omega)\right)} \leqslant C
$$

which is easily obtained from (4.33) and the subsequent comment. Thus, with $\varepsilon=\varepsilon_{0} h^{4 / 3}$,

$$
\left\|u-U_{h}\right\|_{L^{\infty}\left(0, T_{0} ; L^{2}(\Omega)\right)} \leqslant C h^{1 / 3} .
$$

Under the hypotheses $U_{h}(0)=E_{h} u_{0}$ and (**), as well as (4.38),

$$
\begin{aligned}
\lambda \| u- & U_{h} \|_{L^{\infty}\left(0, T_{0} ; L^{2}(\Omega)\right)}^{2} \\
& \leqslant 2\left\|H_{\varepsilon}\left(u^{\varepsilon}\right)-H_{\varepsilon}\left(U_{h}\right)\right\|_{L^{\infty}\left(0, T_{0} ; F\right)}\left\|u^{\varepsilon}-U_{h}\right\|_{L^{\infty}\left(0, T_{0} ; H^{1}(\Omega)\right)}+C \sqrt{\varepsilon} \\
& \leqslant C h[\ln (1 / h)]^{d / 2}+C \sqrt{\varepsilon},
\end{aligned}
$$

where we have used (1.18), (2.4ii), (2.23ii), (3.10i), (4.39), and (4.46i) under the relation $\varepsilon \leqslant \varepsilon_{0} h^{2}$. Thus,

$$
\left\|u-U_{h}\right\|_{L^{\infty}\left(0, T ; L^{2}(\Omega)\right)} \leqslant C[\ln (1 / h)]^{d / 4} \sqrt{h}
$$

with $d=0$ when $\operatorname{dim}(\Omega)=1$ and $d=2$ when $\operatorname{dim}(\Omega) \geqslant 2$.

It is interesting to note that under the regularity hypothesis

$$
\left\|\left[H_{\varepsilon}\left(u^{\varepsilon}\right)\right]_{t}\right\|_{L^{2}(D)} \leqslant C, \quad 0 \leqslant \varepsilon \leqslant \varepsilon_{0},
$$

one can prove first-order convergence by modifying the proof of Theorem 4.2.

THEOREM 4.6. Let $u$ be the solution of (1.8), let $U_{h}$ be the solution of (4.4), and suppose (4.48) is valid. Then for $0 \leqslant \varepsilon \leqslant \varepsilon_{0} h^{2}$, any $\varepsilon_{0}>0$,

$$
\left\|u-U_{h}\right\|_{L^{2}(D)} \leqslant C h .
$$


Under the quasiuniformity assumption (4.2),

$$
\left\|H(u)-H_{\varepsilon}\left(U_{h}\right)\right\|_{L^{\infty}(0, T ; F)} \leqslant C h .
$$

Proof. We shall need a new bound for term (II) in (4.19). We cite the finite element estimate [9], [38]

$$
\left\|\left(T-T_{h}\right) \psi\right\|_{H^{1}(\Omega)} \leqslant C h\|T \psi\|_{H^{2}(\Omega)} \leqslant C h\|\psi\|_{L^{2}(\Omega)}
$$

for all $\psi \in L^{2}(\Omega)$ and replace (4.22) with the new estimate

$$
\begin{aligned}
|(\mathrm{II})| & \leqslant\left\|\left(T-T_{h}\right)\left[\frac{\partial}{\partial t} H_{\varepsilon}\left(u^{\varepsilon}\right)+f\left(u^{\varepsilon}\right)\right]\right\|_{H^{1}(\Omega)} \cdot\left\|H_{\varepsilon}\left(u^{\varepsilon}\right)-H_{\varepsilon}\left(U_{h}\right)\right\|_{F} \\
& \leqslant C h\left\|\frac{\partial}{\partial t} H_{\varepsilon}\left(u^{\varepsilon}\right)+f\left(u^{\varepsilon}\right)\right\|_{L^{2}(\Omega)} \cdot\left(\left\|H_{\varepsilon}\left(u^{\varepsilon}\right)-H_{\varepsilon}\left(U_{h}\right)\right\|_{F_{h}}+C h\right) \\
& \leqslant C h^{2} \eta^{-1}\left(1+\left\|\frac{\partial}{\partial t} H_{\varepsilon}\left(u^{\varepsilon}\right)\right\|_{L^{2}(\Omega)}^{2}\right)+\eta C\left\|H_{\varepsilon}\left(u^{\varepsilon}\right)-H_{\varepsilon}\left(U_{h}\right)\right\|_{F_{h}}^{2},
\end{aligned}
$$

where we have used (4.28), (4.51), and the Cauchy-Schwarz inequality. Bound (4.52) enables us to replace (4.26) with

$$
\begin{aligned}
\left\|H_{\varepsilon}\left(u^{\varepsilon}\right)-H_{\varepsilon}\left(U_{h}\right)\right\|_{L^{\infty}\left(0, T_{0} ; F_{h}\right)}^{2} & \\
& +\int_{0}^{T_{0}}\left(H_{\varepsilon}\left(u^{\varepsilon}\right)-H_{\varepsilon}\left(U_{h}\right), u^{\varepsilon}-U_{h}\right)_{L^{2}(\Omega)} d t \leqslant C h^{2} .
\end{aligned}
$$

Combine (3.10), (4.15) and (4.53) to verify (4.49) and (4.50).

Remark 4.10. If $U_{h}(0)=E_{h} u_{0}$ as in (4.5) and (**) is valid, Theorem 4.6 still holds. The theorem also holds without the quasiuniformity hypothesis if (4.28) is utilized (cf. Remark 4.4). Theorem 4.5, however, does require the quasiuniformity hypothesis. Note that a modified version of $(* *)$ is necessary in the case $H_{0}=H$.

Remark 4.11. Suppose $U_{h}(0)=E_{h} u_{0},(* *)$ is valid, and the hypotheses of Theorem 4.6 hold. Then

$$
\begin{aligned}
\lambda\left\|u-U_{h}\right\|_{L^{\infty}\left(0, T_{0} ; L^{2}(\Omega)\right)}^{2} & \\
& \leqslant 2\left\|H_{\varepsilon}\left(u^{\varepsilon}\right)-H_{\varepsilon}\left(U_{h}\right)\right\|_{L^{\infty}\left(0, T_{0} ; F\right)}\left\|u^{\varepsilon}-U_{h}\right\|_{L^{\infty}\left(0, T_{0} ; H^{1}(\Omega)\right)}+C \sqrt{\varepsilon} \\
& \leqslant C(h+\sqrt{\varepsilon}) \leqslant C h,
\end{aligned}
$$

where we have used (1.18), (2.4ii), (2.23ii), (4.45), and (4.50) as well as (3.10i).

5. Error Estimates for a Discrete-Time Galerkin Scheme. In this section we shall analyze the backward-difference analogue of (4.4).

Definition 5.1. Let $M$ be a positive integer, let $\Delta t=T_{0} / M$ and $t_{n}=n \Delta t$ for $n=0, \ldots, M$, set $g^{n}=g\left(t_{n}\right)$, and define

$$
\left(\partial^{+} g\right)^{n}=\left(g^{n+1}-g^{n}\right) / \Delta t, \quad n=0, \ldots, M-1,
$$

for any function $g$ on $\left\{t_{n}\right\}$. The discrete-time Galerkin approximation of $u\left(\cdot, t_{n}\right)$ is defined to be $U_{h}^{n}$, where $\left\{U_{h}^{n}\right\}_{n=0}^{M} \subseteq M_{h}$ is recursively generated from the nonlinear algebraic relations

$$
\begin{array}{r}
\left(\partial^{+}\left(H_{\varepsilon}\left(U_{h}\right)\right)^{n}, \chi\right)_{L^{2}(\Omega)}+\left(\nabla U_{h}^{n+1}, \nabla \chi\right)_{L^{2}(\Omega)}+\left(f\left(U_{h}^{n+1}\right), \chi\right)_{L^{2}(\Omega)}=0 \\
\text { for all } \chi \in M_{h}, n=0,1, \ldots, M-1,
\end{array}
$$




$$
P_{h} H_{\varepsilon}\left(U_{h}^{0}\right)=P_{h} H_{\varepsilon}\left(u^{\varepsilon}(\cdot, 0)\right)=P_{h} H_{\varepsilon}\left(u_{0}\right) .
$$

The parameter $\varepsilon$ will be determined later. For convenience in formulation, the reader should understand $\left[H_{\varepsilon}\left(U_{h}\right)\right]^{n}$ in (5.1i) to mean $H_{\varepsilon}\left(U_{h}^{n}\right)$.

Remark 5.1. The existence and uniqueness of $U_{h}^{0}$ in (5.1ii) follows from the theory of maximal monotone operators. For $\varepsilon \geqslant 0$, the existence of unique solutions of (5.1i) can be demonstrated by pseudomonotone operator theory [25]. Under assumption $(* *)$ of Remark 4.5, all of our results are valid if we choose the initial function $U_{h}^{0}=E_{h} u_{0}$ instead of (5.1ii); with a modification for $(* *)$ for $H_{0}=H$.

Remark 5.2. The operator $T_{h}$ induces a pointwise relation on $\Omega$ satisfied by the solution of (5.1i). Comparing the latter with (4.6), we obtain

$$
\left[\partial^{+}\left(T_{h} H_{\varepsilon}\left(U_{h}\right)\right)\right]^{n}+U_{h}^{n+1}=-T_{h} f\left(U_{h}^{n+1}\right)+\frac{1}{|\Omega|} \int_{\Omega} U_{h}^{n+1} .
$$

THEOREM 5.1. Let $u$ be the solution of (1.8), and let $\left\{U_{h}^{n}\right\}_{n=0}^{M}$ be the solution sequence of (5.1). For $\varepsilon=\varepsilon_{0} h^{4 / 3}$ and $\Delta t \leqslant c h^{4 / 3}, \varepsilon_{0}$ and c positive constants,

$$
\left[\sum_{n=0}^{M}\left\|u\left(t_{n}\right)-U_{h}^{n}\right\|_{L^{2}(\Omega)}^{2} \Delta t\right]^{1 / 2} \leqslant C h^{2 / 3} .
$$

If the quasiuniformity hypothesis (4.2) holds and $\left\|H_{\varepsilon}\left(U_{h}^{n}\right)\right\|_{L^{2}(\Omega)} \leqslant C$, then

$$
\operatorname{Max}_{n}\left\|H(u)^{n}-H_{\varepsilon}\left(U_{h}^{n}\right)\right\|_{F} \leqslant C h^{2 / 3}
$$

with these choices of $\varepsilon$ and $\Delta t$.

Proof. Noting by (2.3) that

$$
\begin{aligned}
{\left[\partial^{+}\left(T H_{\varepsilon}\left(u^{\varepsilon}\right)\right)\right]^{n}=} & \frac{\partial}{\partial t} T H_{\varepsilon}\left(u^{\varepsilon}\right)\left(t_{n+1}\right)-\frac{1}{\Delta t} \int_{t_{n}}^{t_{n+1}}\left(\tau-t_{n}\right)\left[T H_{\varepsilon}\left(u^{\varepsilon}\right)\right]_{t t}(\tau) d \tau \\
= & -\left(u^{\varepsilon}\right)^{n+1}-\left(T f\left(u^{\varepsilon}\right)\right)^{n+1}+\frac{1}{|\Omega|} \int_{\Omega}\left(u^{\varepsilon}\right)^{n+1} \\
& +\frac{1}{\Delta t} \int_{t_{n}}^{t_{n+1}}\left(\tau-t_{n}\right)\left[\left\{u^{\varepsilon}+T f\left(u^{\varepsilon}\right)\right\}_{t}(\tau)-\frac{1}{|\Omega|} \int_{\Omega} u_{t}^{\varepsilon}(\tau) d x\right] d \tau
\end{aligned}
$$

and combining this relation with (5.2), we obtain

$$
\begin{aligned}
{\left[\partial ^ { + } \left(T _ { h } \left(H_{\varepsilon}\left(u^{\varepsilon}\right)\right.\right.\right.} & \left.\left.\left.-H_{\varepsilon}\left(U_{h}\right)\right)\right)\right]^{n}+\left(u^{\varepsilon}-U_{h}\right)^{n+1} \\
= & -T_{h}\left[f\left(u^{\varepsilon}\right)-f\left(U_{h}\right)\right]^{n+1}+\frac{1}{|\Omega|} \int_{\Omega}\left(u^{\varepsilon}-U_{h}\right)^{n+1}+W_{\varepsilon, n} \\
& +\left(T_{h}-T\right)\left[\left(\partial^{+}\left(H_{\varepsilon}\left(u^{\varepsilon}\right)\right)\right)^{n}+f\left(u^{\varepsilon}\right)^{n+1}\right],
\end{aligned}
$$

where

$$
W(\varepsilon, n)=\frac{1}{\Delta t} \int_{t_{n}}^{t_{n+1}}\left(\tau-t_{n}\right)\left[u^{\varepsilon}(\tau)+\frac{\partial T f\left(u^{\varepsilon}\right)}{\partial t}(\tau)-\frac{1}{|\Omega|} \int_{\Omega} u_{t}^{\varepsilon}(\tau) d x\right] d \tau
$$


Integrate (5.5) against $\left[H_{\varepsilon}\left(u^{\varepsilon}\right)-H_{\varepsilon}\left(U_{h}\right)\right]^{n+1}$ and use the Cauchy-Schwarz inequality to obtain

$$
\begin{aligned}
\frac{1}{2 \Delta t} & \left\{\left\|H_{\varepsilon}\left(u^{\varepsilon}\right)^{n+1}-H_{\varepsilon}\left(U_{h}^{n+1}\right)\right\|_{F_{h}}^{2}-\left\|H_{\varepsilon}\left(u^{\varepsilon}\right)^{n}-H_{\varepsilon}\left(U_{h}^{n}\right)\right\|_{F_{h}}^{2}\right\} \\
& +\left(H_{\varepsilon}\left(u^{\varepsilon}\right)^{n+1}-H_{\varepsilon}\left(U_{h}^{n+1}\right),\left(u^{\varepsilon}\right)^{n+1}-U_{h}^{n+1}\right)_{L^{2}(\Omega)} \\
\leqslant & -\left(T_{h}\left[f\left(u^{\varepsilon}\right)-f\left(U_{h}\right)\right]^{n+1}, H_{\varepsilon}\left(u^{\varepsilon}\right)^{n+1}-H_{\varepsilon}\left(U_{h}^{n+1}\right)\right)_{L^{2}(\Omega)} \\
& +\left(\left(T_{h}-T\right)\left[\left(\partial^{+} H_{\varepsilon}\left(u^{\varepsilon}\right)\right)^{n}+f\left(u^{\varepsilon}\right)^{n+1}\right], H_{\varepsilon}\left(u^{\varepsilon}\right)^{n+1}-H_{\varepsilon}\left(U_{h}^{n+1}\right)\right)_{L^{2}(\Omega)} \\
& +\frac{1}{|\Omega|} \int_{\Omega}\left(u^{\varepsilon}-U_{h}\right)^{n+1} \int_{\Omega}\left[H_{\varepsilon}\left(u^{\varepsilon}\right)-H_{\varepsilon}\left(U_{h}\right)\right]^{n+1} \\
& +\left(W_{\varepsilon, n}, H_{\varepsilon}\left(u^{\varepsilon}\right)^{n+1}-H_{\varepsilon}\left(U_{h}^{n+1}\right)\right)_{L^{2}(\Omega)} \\
= & (\mathrm{I})_{n+1}+(\mathrm{II})_{n+1}+(\mathrm{III})_{n+1}+(\mathrm{IV})_{n+1} .
\end{aligned}
$$

We shall first estimate the term (IV) $)_{n+1}$. We have

$$
(\mathrm{IV})_{n+1} \leqslant \frac{1}{2 \eta \varepsilon}\left\|W_{\varepsilon, n}\right\|_{L^{2}(\Omega)}^{2}+\frac{1}{2} \eta \varepsilon\left\|H_{\varepsilon}\left(u^{\varepsilon}\right)^{n+1}-H_{\varepsilon}\left(U_{h}^{n+1}\right)\right\|_{L^{2}(\Omega)}^{2},
$$

and the choice $\eta=1 /(4 \gamma)$, coupled with (4.21), permits the second term on the right-hand side of (5.7) to be absorbed on the left-hand side of (5.6). In estimating the first term on the right-hand side of (5.7), we shall interchange $T$ and $\partial / \partial t$ and use the inequality

$$
\left\|\frac{\partial f\left(u^{\varepsilon}\right)}{\partial t}\right\|_{L^{2}(\Omega)} \leqslant C\left\|u_{t}^{\varepsilon}\right\|_{L^{2}(\Omega)}
$$

where $C$ is a Lipschitz constant for $f$, to obtain

$$
\left\|W_{\varepsilon, n}\right\|_{L^{2}(\Omega)} \leqslant C \sqrt{\Delta t}\left\|u_{t}^{\varepsilon}\right\|_{L^{2}\left(t_{n}, t_{n+1} ; L^{2}(\Omega)\right)}
$$

for some constant $C$. Here we have also used the triangle inequality and the Cauchy-Schwarz inequality. We now bound (I $)_{n+1},(\text { II })_{n+1}$, (III $)_{n+1}$ in a manner virtually identical to the corresponding quantities (I), (II), and (III) in the proof of Theorem 4.2 , replacing $1 / 3$ with $1 / 4$ and $1 / 6$ with $1 / 8$. This yields

$$
\begin{aligned}
& \frac{1}{2 \Delta t}\left\{\left\|H_{\varepsilon}\left(u^{\varepsilon}\right)^{n+1}-H_{\varepsilon}\left(U_{h}^{n+1}\right)\right\|_{F_{h}}^{2}-\left\|H_{\varepsilon}\left(u^{\varepsilon}\right)^{n}-H_{\varepsilon}\left(U_{h}^{n}\right)\right\|_{F_{h}}^{2}\right\} \\
& +\frac{1}{2}\left(H_{\varepsilon}\left(u^{\varepsilon}\right)^{n+1}-H_{\varepsilon}\left(U_{h}^{n+1}\right),\left(u^{\varepsilon}-U_{h}\right)^{n+1}\right)_{L^{2}(\Omega)} \\
& \leqslant C\left\{\frac{h^{4}}{\varepsilon}\left(\left\|\left[\partial^{+} H_{\varepsilon}\left(u^{\varepsilon}\right)\right]^{n}\right\|_{L^{2}(\Omega)}^{2}+\left\|\left(u^{\varepsilon}\right)^{n+1}\right\|_{L^{2}(\Omega)}^{2}\right)\right. \\
& \left.\quad+\frac{\Delta t}{\varepsilon}\left\|u_{t}^{\varepsilon}\right\|_{L^{2}\left(t_{n}, t_{n+1} ; L^{2}(\Omega)\right)}^{2}+\left\|H_{\varepsilon}\left(u^{\varepsilon}\right)^{n+1}-H_{\varepsilon}\left(U_{h}^{n+1}\right)\right\|_{F_{h}}^{2}\right\} .
\end{aligned}
$$

In order to put this in a form suitable for an application of the discrete Gronwall inequality, we estimate the first term on the right-hand side of (5.9). This yields

$$
\begin{aligned}
& \left\|\left[\partial^{+} H_{\varepsilon}\left(u^{\varepsilon}\right)\right]^{n}\right\|_{L^{2}(\Omega)}=\left\|\frac{1}{\Delta t} \int_{t_{n}}^{t_{n+1}}\left[H_{\varepsilon}\left(u^{\varepsilon}\right)\right]_{t}(\tau) d \tau\right\|_{L^{2}(\Omega)} \\
& \quad \leqslant \frac{1}{\Delta t} \int_{t_{n}}^{t_{n+1}}\left\|\left[H_{\varepsilon}\left(u^{\varepsilon}\right)\right]_{t}(\tau)\right\|_{L^{2}(\Omega)} d \tau \leqslant \frac{C}{\sqrt{\Delta t}}\left\|\left[H_{\varepsilon}\left(u^{\varepsilon}\right)\right]_{t}\right\|_{L^{2}\left(t_{n}, t_{n+1} ; L^{2}(\Omega)\right)} .
\end{aligned}
$$


Combining (5.9) and (5.10), multiplying through by $\Delta t$, and summing on $n$ from $n=0$ to $n=m-1,0<m \leqslant M$, gives

$$
\begin{aligned}
\frac{1}{2}\left\{\left\|H_{\varepsilon}\left(u^{\varepsilon}\right)^{m}-H_{\varepsilon}\left(U_{h}^{m}\right)\right\|_{F_{h}}^{2}-\left\|H_{\varepsilon}\left(u^{\varepsilon}\right)^{0}-H_{\varepsilon}\left(U_{h}^{0}\right)\right\|_{F_{h}}^{2}\right\} \\
+\frac{1}{2} \sum_{n=0}^{m-1}\left(H_{\varepsilon}\left(u^{\varepsilon}\right)^{n+1}-H_{\varepsilon}\left(U_{h}^{n+1}\right),\left(u^{\varepsilon}\right)^{n+1}-U_{h}^{n+1}\right)_{L^{2}(\Omega)} \Delta t \\
\leqslant C\left\{\frac{h^{4}}{\varepsilon}\left(\left\|\left[H_{\varepsilon}\left(u^{\varepsilon}\right)\right]_{t}\right\|_{L^{2}(D)}^{2}+C\right)+\frac{(\Delta t)^{2}}{\varepsilon}\left\|u_{t}^{\varepsilon}\right\|_{L^{2}(D)}^{2}\right. \\
\left.+\sum_{n=0}^{m-1}\left\|H_{\varepsilon}\left(u^{\varepsilon}\right)^{n+1}-H_{\varepsilon}\left(U_{h}^{n+1}\right)\right\|_{F_{h}}^{2} \Delta t\right\} .
\end{aligned}
$$

Applying the discrete Gronwall inequality to (5.11) yields, for some $\eta>0$,

$$
\begin{gathered}
\operatorname{Max}_{0 \leqslant n \leqslant M}\left\|H_{\varepsilon}\left(u^{\varepsilon}\right)^{n}-H_{\varepsilon}\left(U_{h}^{n}\right)\right\|_{F_{h}}^{2}+\eta \sum_{n=0}^{M}\left(H_{\varepsilon}\left(u^{\varepsilon}\right)^{n}-H_{\varepsilon}\left(U_{h}^{n}\right),\left(u^{\varepsilon}\right)^{n}-U_{h}^{n}\right) \Delta t \\
\leqslant C\left\{\frac{h^{4}}{\varepsilon}\left(\left\|\left[H_{\varepsilon}\left(u^{\varepsilon}\right)\right]_{t}\right\|_{L^{2}(D)}^{2}+C\right)+\frac{(\Delta t)^{2}}{\varepsilon}\left\|u_{t}^{\varepsilon}\right\|_{L^{2}(D)}^{2}\right\}
\end{gathered}
$$

Using (2.4ii, iii), we see that the right-hand side of (5.12) is bounded above by $C\left(h^{4} / \varepsilon^{2}+(\Delta t)^{2} / \varepsilon\right)$. Comparing this bound with that given in Theorem 3.3, estimate (3.19), i.e., with the identification $\left(u^{\varepsilon}\right)^{n}=u^{\varepsilon n}$,

$$
\begin{aligned}
\left.\underset{0 \leqslant n \leqslant M}{\operatorname{Max}_{0 \leqslant n} \|\left[H_{\varepsilon}\left(u^{\varepsilon}\right)\right.}-H(u)\right]^{n}\left\|_{F}^{2}+\sum_{n=0}^{M}\right\|\left(u^{\varepsilon}\right)^{n}-u^{n} \|_{L^{2}(\Omega)}^{2} \Delta t \\
\leqslant C\left(\varepsilon+\left((\Delta t)^{2} / \varepsilon\right)\right),
\end{aligned}
$$

we see that setting $\varepsilon=\varepsilon_{0} h^{4 / 3}$ and choosing $\Delta t \leqslant c h^{4 / 3}$ for some positive constants $\varepsilon_{0}$ and $c$ yields (5.3).

To verify (5.4) we assume the quasiuniformity hypothesis (4.2); by (4.15ii) we may rewrite (5.12) as

$$
\begin{aligned}
& \delta^{2} \operatorname{Max}_{0 \leqslant n \leqslant M}\left\|P_{h}\left[H_{\varepsilon}\left(u^{\varepsilon}\right)^{n}-H_{\varepsilon}\left(U_{h}^{n}\right)\right]\right\|_{F}^{2} \\
& \quad+\eta \sum_{n=0}^{M}\left(H_{\varepsilon}\left(u^{\varepsilon}\right)^{n}-H_{\varepsilon}\left(U_{h}^{n}\right),\left(u^{\varepsilon}\right)^{n}-U_{h}^{n}\right)_{L^{2}(\Omega)} \Delta t \leqslant C\left(\frac{h^{4}}{\varepsilon^{2}}+\frac{(\Delta t)^{2}}{\varepsilon}\right) .
\end{aligned}
$$

Comparing (5.13) and (5.14), we see that it remains to prove

$$
\operatorname{Max}_{0 \leqslant n \leqslant M}\left\|\left(I-P_{h}\right)\left[H_{\varepsilon}\left(u^{\varepsilon}\right)^{n}-H_{\varepsilon}\left(U_{h}^{n}\right)\right]\right\|_{F} \leqslant C h .
$$

By an inequality similar to (4.31), it suffices to know that, for $n=0, \ldots, M$,

$$
\begin{aligned}
& \text { (i) }\left\|H_{\varepsilon}\left(u^{\varepsilon}\right)^{n}\right\|_{L^{2}(\Omega)} \leqslant C, \\
& \text { (ii) }\left\|H_{\varepsilon}\left(U_{h}^{n}\right)\right\|_{L^{2}(\Omega)} \leqslant C .
\end{aligned}
$$

However, (5.16i) follows from

$$
\left\|H_{\varepsilon}\left(u^{\varepsilon}\right)\right\|_{L^{2}(\Omega)} \leqslant\left\|H_{\varepsilon}\left(u^{\varepsilon}\right)\right\|_{L^{\infty}\left(0, T_{0} ; L^{2}(\Omega)\right)} \leqslant C,
$$


where the latter inequality makes use of (2.4i) sharpened by the continuity of $H_{\varepsilon}\left(u^{\varepsilon}\right)$. (5.16ii) is the finite element analogue of (2.4i) and the fully discrete analogue of (4.32); its validity is an assumption of Theorem 5.1. This establishes (5.15) and hence (5.4).

Remark 5.3. The quasiuniformity hypothesis in Theorem 5.1 is unnecessary if use is made of (4.28) with $f=\left[H_{\varepsilon}\left(u^{\varepsilon}\right)-H_{\varepsilon}\left(U_{h}\right)\right]^{n}$. The constraint $\Delta t \leqslant c \cdot h^{4 / 3}$ in Theorem 5.1 is a bit surprising; one might expect the usual constraint $\Delta t \leqslant \mathrm{c} \cdot h^{2}$ to be necessary to maintain the global convergence rate. We get the latter in our next result, because the analysis of (5.1) with $\varepsilon=0$ requires a cruder estimate of term $(\text { IV })_{n+1}$.

THEOREM 5.2. Suppose that the quasiuniformity assumption (4.2) holds. Assume that the solution of (5.1) satisfies

$$
\operatorname{Max}_{0 \leqslant n \leqslant M}\left\|H_{\varepsilon}\left(U_{h}^{n}\right)\right\|_{L^{\infty}(\Omega)} \leqslant C \quad(C \text { ind } \text { of } \varepsilon, h)
$$

for $0 \leqslant \varepsilon \leqslant \varepsilon_{0} h^{2}$. Then, with $\Delta t \leqslant c \cdot h^{2}$, and $h \leqslant 1$,

$$
\operatorname{Max}_{0 \leqslant n \leqslant M}\left\|H(u)^{n}-H_{\varepsilon}\left(U_{h}^{n}\right)\right\|_{F} \leqslant \begin{cases}C h, & \operatorname{dim}(\Omega)=1, \\ C(\ln (1 / h)) h, & \operatorname{dim}(\Omega) \geqslant 2,\end{cases}
$$

and

$$
\left(\sum_{n}\left\|u^{n}-U_{h}^{n}\right\|_{L^{2}(\Omega)}^{2} \Delta t\right)^{1 / 2} \leqslant \begin{cases}C h, & \operatorname{dim}(\Omega)=1 \\ C(\ln (1 / h)) h, & \operatorname{dim}(\Omega) \geqslant 2 .\end{cases}
$$

Proof. Returning to inequality (5.6), we bound the terms (I) $)_{n+1}$ and (III) $)_{n+1}$ as previously. We also bound the term in (II) $)_{n+1}$ involving $\left(T_{h}-T\right) f\left(u^{\varepsilon}\right)^{n+1}$ as in (4.52). A different duality estimate is required for the remaining term. We have

$$
\begin{aligned}
\left|\left(\mathrm{II}^{\prime}\right)_{n+1}\right| & =\left|\left(\left(T_{h}-T\right)\left(\partial^{+} H_{\varepsilon}\left(u^{\varepsilon}\right)\right)^{n}, H_{\varepsilon}\left(u^{\varepsilon}\right)^{n+1}-H_{\varepsilon}\left(U_{h}^{n+1}\right)\right)_{L^{2}(\Omega)}\right| \\
& \leqslant\left\|\left(T_{h}-T\right)\left(\partial^{+} H_{\varepsilon}\left(u^{\varepsilon}\right)\right)^{n}\right\|_{M(\bar{\Omega})}\left\|H_{\varepsilon}\left(u^{\varepsilon}\right)^{n+1}-H_{\varepsilon}\left(U_{h}^{n+1}\right)\right\|_{L^{\infty}(\Omega)} \\
& \leqslant C\left\|\left(T_{h}-T\right)\left(\partial^{+} H_{\varepsilon}\left(u^{\varepsilon}\right)\right)^{n}\right\|_{M(\bar{\Omega})},
\end{aligned}
$$

where we have used (2.23i), (4.34), and (5.17). Thus,

$$
\left|\left(\mathrm{II}^{\prime}\right)_{n+1}\right| \leqslant\left\|\frac{1}{\Delta t} \int_{t_{n}}^{t_{n+1}}\left(T_{h}-T\right) \frac{\partial}{\partial t} H_{\varepsilon}\left(u^{\varepsilon}\right)(\tau) d \tau\right\|_{M(\bar{\Omega})},
$$

where the interchange of $\left(T_{h}-T\right)$ and $\partial / \partial t$ may be justified by using (1.10)-(1.11), (4.6)-(4.7),

$$
\begin{aligned}
& \leqslant C \frac{1}{\Delta t} \int_{t_{n}}^{t_{n+1}}\left\|\left(T_{h}-T\right) \frac{\partial}{\partial t} H_{\varepsilon}\left(u^{\varepsilon}\right)(\tau)\right\|_{M(\bar{\Omega})} d \tau \\
& \leqslant C\left\|\left(T_{h}-T\right) \frac{\partial}{\partial t} H_{\varepsilon}\left(u^{\varepsilon}\right)\right\|_{L^{\infty}\left(t_{n}, t_{n+1} ; M(\bar{\Omega})\right)},
\end{aligned}
$$

and the latter quantity may be estimated as in estimating (4.41). Thus, term $\left(\mathrm{II}^{\prime}\right)_{n+1}$ is $O\left(h^{2}\right)$ in the one-dimensional case and $O\left([\ln (1 / h)]^{2} h^{2}\right)$ when $\operatorname{dim}(\Omega)$ is $\geqslant 2$. 
Next, we must treat term (IV) $)_{n+1}$. We have

$$
\begin{aligned}
\left|(\mathrm{IV})_{n+1}\right| \leqslant & \left\|\frac{1}{\Delta t} \int_{t_{n}}^{t_{n+1}}\left(\tau-t_{n}\right)\left[u_{t}^{\varepsilon}(\tau)+\frac{\partial}{\partial t} T f\left(u^{\varepsilon}\right)(\tau)-\frac{1}{|\Omega|} \int_{\Omega} u_{t}^{\varepsilon}(\tau) d x\right] d \tau\right\|_{L^{\prime}(\Omega)} \\
& \times\left\|H_{\varepsilon}\left(u^{\varepsilon}\right)^{n+1}-H_{\varepsilon}\left(U_{h}^{n+1}\right)\right\|_{L^{\infty}(\Omega)} \\
\leqslant & \frac{C}{\Delta t} \int_{t_{n}}^{t_{n+1}}\left(\tau-t_{n}\right)\left\|u_{t}^{\varepsilon}(\tau)\right\|_{L^{\prime}(\Omega)} d \tau,
\end{aligned}
$$

where we have used (2.23i), (5.17) and

$$
\left\|\frac{\partial f\left(u^{\varepsilon}\right)}{\partial t}\right\|_{L^{\prime}(\Omega)} \leqslant C\left\|\frac{\partial u^{\varepsilon}}{\partial t}\right\|_{L^{\prime}(\Omega)} .
$$

This gives

$$
\left|(\mathrm{IV})_{n+1}\right| \leqslant C \Delta t\left\|u_{t}^{\varepsilon}\right\|_{L^{\infty}\left(t_{n}, t_{n+1} ; L^{1}(\Omega)\right)}
$$

Combining these reestimations with (5.6) and the previous bounds gives

$$
\begin{gathered}
\frac{1}{2 \Delta t}\left\{\left\|H_{\varepsilon}\left(u^{\varepsilon}\right)^{n+1}-H_{\varepsilon}\left(U_{h}^{n+1}\right)\right\|_{F_{h}}^{2}-\left\|H_{\varepsilon}\left(u^{\varepsilon}\right)^{n}-H_{\varepsilon}\left(U_{h}^{n}\right)\right\|_{F_{h}}^{2}\right\} \\
+\frac{1}{2}\left(H_{\varepsilon}\left(u^{\varepsilon}\right)^{n+1}-H_{\varepsilon}\left(U_{h}^{n+1}\right),\left(u^{\varepsilon}\right)^{n+1}-U_{h}^{n+1}\right)_{L^{2}(\Omega)} \\
\leqslant C\left\{\left\|H_{\varepsilon}\left(u^{\varepsilon}\right)^{n+1}-H_{\varepsilon}\left(U_{h}^{n+1}\right)\right\|_{F_{h}}^{2}\right. \\
+[\ln (1 / h)]^{d} h^{2}\left\|\left[H_{\varepsilon}\left(u^{\varepsilon}\right)\right]_{t}\right\|_{L^{\infty}\left(t_{n}, t_{n+1} ; M(\bar{\Omega})\right)} \\
\left.+\Delta t\left\|_{t}^{\varepsilon}\right\|_{L^{\infty}\left(t_{n}, t_{n+1} ; L^{1}(\Omega)\right)}\right\}+C h^{2}
\end{gathered}
$$

where $d=0$ or 2 according as $\operatorname{dim}(\Omega)=1$ or $\operatorname{dim}(\Omega) \geqslant 2$. Multiply (5.21) by $\Delta t$, sum on $n$, and use the discrete Gronwall lemma to obtain

$$
\begin{aligned}
& \underset{0 \leqslant n \leqslant M}{\operatorname{Max}_{0 \leqslant} \| H_{\varepsilon}\left(u^{\varepsilon}\right)^{n}}-H_{\varepsilon}\left(U_{h}^{n}\right) \|_{F_{h}}^{2} \\
&+\eta \sum_{n=0}^{M}\left(H_{\varepsilon}\left(u^{\varepsilon}\right)^{n}-H_{\varepsilon}\left(U_{h}^{n}\right),\left(u^{\varepsilon}\right)^{n}-U_{h}^{n}\right)_{L^{2}(\Omega)} \Delta t \\
& \leqslant C[\ln (1 / h)]^{d} h^{2}\left\|\left[H_{\varepsilon}\left(u^{\varepsilon}\right)\right]_{t}\right\|_{L^{\infty}\left(0, T_{0} ; M(\bar{\Omega})\right)} \\
&+C \cdot \Delta t \cdot\left\|u_{t}^{\varepsilon}\right\|_{L^{\infty}\left(0, T_{0} ; L^{1}(\Omega)\right)}+C h^{2} \\
& \leqslant C\left\{[\ln (1 / h)]^{d} h^{2}+\Delta t\right\}
\end{aligned}
$$

where we have used (2.6) and (2.22). Comparison of (5.22) and estimate (3.20) of Theorem 3.3 yields (5.19). Use (3.20), (4.15ii), (5.15) and (5.22) to obtain (5.18).

Remark 5.4. It is not possible to remove the quasiuniformity hypothesis in Theorem 5.2. However, the technique discussed in Remark 5.3 is valid in Theorem 5.3 to follow. The argument used to derive (4.46) may be used to show that, under the hypotheses of Theorem 5.1, with $U_{h}^{0}=E_{h} u_{0}$ substituted and (**) of Remark 4.5 assumed, and with a modification in (**) in the case $H_{0}=H$,

$$
\operatorname{Max}_{n}\left\|u^{n}-U_{h}^{n}\right\|_{L^{2}(\Omega)} \leqslant C h^{1 / 3} \text {. }
$$


Under the hypotheses of Theorem 5.2, the argument used to derive (4.47) may be used to obtain

$$
\operatorname{Max}_{n}\left\|u^{n}-U_{h}^{n}\right\|_{L^{2}(\Omega)} \leqslant C[\ln (1 / h)]^{d / 4} \sqrt{h}
$$

where $d=0$ if $\operatorname{dim}(\Omega)=1$ and $d=2$ if $\operatorname{dim}(\Omega) \geqslant 2$.

It is interesting to see how our convergence estimates can be strengthened if we make some assumptions concerning the regularity of the solution of the Stefan problem (2.1).

THEOREM 5.3. Suppose (4.48) and the hypotheses of Theorem 5.1 hold. Then, for $\varepsilon=\varepsilon_{0} h^{2}$ and $\Delta t \leqslant c h^{2}, \varepsilon_{0}$ and c positive constants,

$$
\left[\sum_{n=0}^{M}\left\|u\left(t_{n}\right)-U_{h}^{n}\right\|_{L^{2}(\Omega)}^{2} \cdot \Delta t\right]^{1 / 2} \leqslant C h .
$$

Under the quasiuniformity assumption (4.2), we also have

$$
\underset{n}{\operatorname{Max}}\left\|H(u)^{n}-H_{\varepsilon}\left(U_{h}^{n}\right)\right\|_{F} \leqslant C h .
$$

If the regularity hypothesis

$$
\left\|\nabla u_{t}^{\varepsilon}\right\|_{L^{2}(D)} \leqslant C, \quad 0 \leqslant \varepsilon \leqslant \varepsilon_{0},
$$

is valid, then we need only assume that $0 \leqslant \varepsilon \leqslant \varepsilon_{0} h^{2}$ and $\Delta t \leqslant c h$ to obtain (5.25) and (5.26) provided, for the latter, (3.10i) holds uniformly in $t$.

Proof. We need a new bound for term (II) $)_{n+1}$ in (5.6). By (5.16) and (4.28),

$$
\begin{aligned}
\left|(\mathrm{II})_{n+1}\right| \leqslant & \left\|\left(T_{h}-T\right)\left[\left(\partial^{+} H_{\varepsilon}\left(u^{\varepsilon}\right)\right)^{n}+f\left(u^{\varepsilon}\right)^{n+1}\right]\right\|_{H^{1}(\Omega)} \\
& \cdot\left\|H_{\varepsilon}\left(u^{\varepsilon}\right)^{n+1}-H_{\varepsilon}\left(U_{h}^{n+1}\right)\right\|_{F} \\
\leqslant & \frac{1}{2}\left\|\left(T_{h}-T\right)\left[\left(\partial^{+} H_{\varepsilon}\left(u^{\varepsilon}\right)\right)^{n}+f\left(u^{\varepsilon}\right)^{n+1}\right]\right\|_{H^{1}(\Omega)}^{2} \\
& +\frac{1}{2}\left\|H_{\varepsilon}\left(u^{\varepsilon}\right)^{n+1}-H_{\varepsilon}\left(U_{h}^{n+1}\right)\right\|_{F_{h}}^{2}+C h^{2} .
\end{aligned}
$$

Use (2.4i) and the finite element estimate [9], [38]

$$
\left\|\left(T-T_{h}\right) \phi\right\|_{H^{1}(\Omega)} \leqslant C h\|T \phi\|_{H^{2}(\Omega)} \leqslant C h\|\phi\|_{L^{2}(\Omega)}, \quad \phi \in L^{2}(\Omega),
$$

to see that

$$
\begin{aligned}
& \text { (5.30) }\left\|\left(T_{h}-T\right)\left[\left(\partial^{+} H_{\varepsilon}\left(u^{\varepsilon}\right)\right)^{n}-f\left(u^{\varepsilon}\right)^{n+1}\right]\right\|_{H^{\prime}(\Omega)} \\
& \leqslant C h\left\|\left(\partial^{+} H_{\varepsilon}\left(u^{\varepsilon}\right)\right)^{n}-f\left(u^{\varepsilon}\right)^{n+1}\right\|_{L^{2}(\Omega)} \\
& \leqslant C h\left\{1+\frac{1}{\Delta t} \int_{t_{n}}^{t_{n+1}}\left\|\left[H_{\varepsilon}\left(u^{\varepsilon}\right)\right]_{t}(\tau)\right\|_{L^{2}(\Omega)} d \tau\right\} \\
& \leqslant C h\left\{1+\frac{1}{\sqrt{\Delta t}}\left\|\left[H_{\varepsilon}\left(u^{\varepsilon}\right)\right]_{t}(\tau)\right\|_{L^{2}\left(t_{n}, t_{n+1} ; L^{2}(\Omega)\right)}\right\} \text {. }
\end{aligned}
$$


Estimates (5.28) and (5.30) allow us to replace (5.12) by

$$
\begin{aligned}
\underset{n}{\operatorname{Max}_{n} \| H_{\varepsilon}\left(u^{\varepsilon}\right)^{n}}- & H_{\varepsilon}\left(U_{h}^{n}\right) \|_{F_{h}}^{2} \\
& +\eta \sum_{n=0}^{M}\left(H_{\varepsilon}\left(u^{\varepsilon}\right)^{n}-H_{\varepsilon}\left(U_{h}^{n}\right),\left(u^{\varepsilon}\right)^{n}-U_{h}^{n}\right)_{L^{2}(\Omega)} \cdot \Delta t \\
\leqslant & C\left\{h^{2}\left(\left\|\left[H_{\varepsilon}\left(u^{\varepsilon}\right)\right]_{t}\right\|_{L^{2}(D)}^{2}+1\right)+\frac{(\Delta t)^{2}}{\varepsilon}\left\|u_{t}^{\varepsilon}\right\|_{L^{2}(D)}^{2}\right\} \\
\leqslant & C\left(h^{2}+\frac{(\Delta t)^{2}}{\varepsilon}\right),
\end{aligned}
$$

where we have used (2.4ii) and (4.48). Compare (3.19) and (5.31) to obtain (5.25), and also use (5.15) to obtain (5.26) with $\varepsilon=\varepsilon_{0} h^{2}$ and $\Delta t \leqslant c h^{2}$.

Next, we will derive a new estimate for term (IV) $)_{n+1}$ in (5.6) in order to take advantage of (5.27). By (4.28) and (5.16),

$$
\begin{aligned}
& |(\mathrm{IV})|_{n+1} \leqslant\left\|W_{\varepsilon, n}\right\|_{H^{1}(\Omega)}\left\|H_{\varepsilon}\left(u^{\varepsilon}\right)^{n+1}-H_{\varepsilon}\left(U_{h}^{n+1}\right)\right\|_{F} \\
& \leqslant \frac{1}{2}\left\|\frac{1}{\Delta t} \int_{t_{n}}^{t_{n+1}}\left(\tau-t_{n}\right)\left[u_{t}^{\varepsilon}(\tau)+\frac{\partial}{\partial t} T f\left(u^{\varepsilon}\right)(\tau)-\frac{1}{|\Omega|} \int_{\Omega} u_{t}^{\varepsilon}(\tau)\right]\right\|_{H^{\prime}(\Omega)}^{2} \\
& \quad+\frac{1}{2}\left\|H_{\varepsilon}\left(u^{\varepsilon}\right)^{n+1}-H_{\varepsilon}\left(U_{h}^{n+1}\right)\right\|_{F_{h}}^{2}+C h^{2}
\end{aligned}
$$

By the above

$$
\begin{aligned}
\left\|W_{\varepsilon, n}\right\|_{H^{1}(\Omega)} & \leqslant \frac{C}{\Delta t}\left(\int_{t_{n}}^{t_{n+1}}\left(\tau-t_{n}\right)\left(\left\|u_{t}^{\varepsilon}(\tau)\right\|_{H^{1}(\Omega)}\right) d \tau\right) \\
& \leqslant C \cdot \sqrt{\Delta t} \cdot\left(\left\|u_{t}^{\varepsilon}\right\|_{L^{2}\left(t_{n}, t_{n+1} ; H^{1}(\Omega)\right)}\right) .
\end{aligned}
$$

Use (5.32) and (5.33) to replace (5.31) by

$$
\begin{aligned}
\underset{0 \leqslant n \leqslant M}{\operatorname{Max}} \| H_{\varepsilon}\left(u^{\varepsilon}\right)^{n}- & H_{\varepsilon}\left(U_{h}^{n}\right) \|_{F_{h}}^{2} \\
& +\eta \sum_{n=0}^{M}\left(H_{\varepsilon}\left(u^{\varepsilon}\right)^{n}-H_{\varepsilon}\left(U_{h}^{n}\right),\left(u^{\varepsilon}\right)^{n}-U_{h}^{n}\right)_{L^{2}(\Omega)} \cdot \Delta t \\
& \leqslant C\left\{h^{2}\left(\left\|\left[H_{\varepsilon}\left(u^{\varepsilon}\right)\right]_{t}\right\|_{L^{2}(D)}^{2}+1\right)+h^{2}+(\Delta t)^{2}\right\} \\
& \leqslant C\left(h^{2}+(\Delta t)^{2}\right)
\end{aligned}
$$

where we have used (4.48) and (5.27). Compare (3.10ii) and (5.34) to establish (5.25) with $0 \leqslant \varepsilon \leqslant \varepsilon_{0} h^{2}$ and $\Delta t \leqslant c h$; (5.26) follows as above from (3.10i).

Remark 5.4. The rates (5.25)-(5.26) for $0 \leqslant \varepsilon \leqslant \varepsilon_{0} h^{2}$ and $\Delta t \leqslant c h$ appear to be in good agreement with numerical experience for the scheme (5.1) [41].

Remark 5.5. If $(* *)$ is true and we use the initial datum $U_{h}^{0}=E_{h} u_{0}$, then, under the hypotheses of Theorem 5.3,

$$
\left\|u-U_{h}\right\|_{L^{\infty}\left(0, T_{0} ; L^{2}(\Omega)\right)} \leqslant C \sqrt{h} .
$$

The same estimate also holds with the choice dictated by (5.1ii). 
Remark 5.6. If we assume (5.27) and the hypotheses of Theorem 5.2, then

$$
\left\|u-U_{h, s}\right\|_{L^{\infty}\left(0, T_{0} ; L^{2}(\Omega)\right)} \leqslant C[\log (1 / h)]^{d / 4} \sqrt{h},
$$

where $d=0$ if $\operatorname{dim}(\Omega)=1, d=2$ if $\operatorname{dim}(\Omega)=2$ or $3,0 \leqslant \varepsilon \leqslant \varepsilon_{0} h^{2}$, and $\Delta t \leqslant c h$, and $U_{h, s}$ is the step-function with value $U_{h}^{n}$ on $t_{n} \leqslant t \leqslant t_{n+1}$.

6. Conclusions. The Stefan problem (1.1) is similar to a problem discussed in Wheeler's paper [40]. Wheeler considers the problem (1.2), instead of the transformed version (1.1), and assigns the boundary data

$$
\begin{array}{rlrl}
\theta & =b(x, t) & & \text { on } \partial \Omega_{1} \times\left(0, T_{0}\right], \\
k \frac{\partial \theta}{\partial \nu} & =q(x, t, \theta) & \text { on } \partial \Omega_{2} \times\left(0, T_{0}\right],
\end{array}
$$

where $\partial \Omega$ is the disjoint union of $\partial \Omega_{1}$ and $\partial \Omega_{2}$; our notation is as in Section 1 . Provided $b$ and $q$ are $C^{1}$ functions of their arguments and that $q$ is linear in $\theta$, the error analysis of this paper can be extended with minor modifications to treat (1.2)-(6.1) with the initial temperature

$$
\begin{aligned}
& \theta(\cdot, 0)=\theta_{0}(\cdot) \in H^{2}(\Omega), \\
& \sigma\left(\theta_{0}\right)+s\left(\theta_{0}\right) \in L^{\infty}(\Omega) .
\end{aligned}
$$

Wheeler computes the integrals over $\Omega$ exactly in the nonlinear algebraic equations corresponding to (5.1). He then uses a constrained Newtonian iteration scheme to approximate the solution of the algebraic problems. The scheme was found to converge decently with $\varepsilon=0$ but setting $\varepsilon>0$ accelerated the convergence.

The error analysis of the scheme (5.1) is of interest chiefly in a neighborhood of the phase transition region $\{(x, t): u(x, t)=0\}$. Away from the front (1.li) becomes the heat equation, $u(x, t)$ is smooth [23], and one would expect an optimal-order $O\left(h^{2}+\Delta t\right)$ local $L^{2}$ convergence rate. This suggests that for the $\varepsilon=0$ scheme a coarse triangulation with mesh size $h_{c}$ proportional to $\sqrt{h_{f}}$ be used away from the front, where $h_{f}$ is the fine mesh spacing employed near the phase transition. The time step size may be chosen as in Theorem 5.2 to maintain an overall $O\left(h_{f}\right)$ convergence rate.

Acknowledgements. The authors benefitted greatly from the physical insight and computational experience of John A. Wheeler, Jr. of Exxon Production Research Company. Special thanks are due to the referee for a careful reading and several helpful literature references, and to Lars Wahlbin for outlining the argument leading to (4.35ii).

\footnotetext{
Department of Mathematics

Northwestern University

Evanston, Illinois 60201

Department of Mathematics

Tulane University

New Orleans, Louisiana 70118
}

1. S. Agmon, A. Douglas \& L. Nirenberg, "Estimates near the boundary for solutions of elliptic partial differential equations satisfying general boundary conditions," Comm. Pure Appl. Math., v. 12, 1959 , pp. $623-727$. 
2. O. B. ANDERSLAND \& D. M. ANDERSON (eds.), Geotechnical Engineering for Cold Regions, McGraw-Hill, New York, 1978.

3. D. M. ANDERSON \& N. R. MORgenstern, "Physics, chemistry and mechanics of frozen ground," in Proc. North American Permafrost Second International Conf., Nat. Acad. Sciences, Washington, D. C., 1973, pp. 257-295.

4. J. H. Bramble, A. H. Schatz, V. Thomee \& L. B. Wahlbin, "Some convergence estimates for semi-discrete Galerkin type approximations for parabolic equations," SIAM J. Numer. Anal., v. 14, 1977, pp. $218-241$.

5. H. Brezis, Operateurs Maximaux Monotones et Semi-groupes de Contractions dans les Espaces de Hilbert, North-Holland/American Elsevier, Amsterdam/New York, 1973.

6. H. Brezis \& W. Strauss, "Semilinear elliptic equations in $L^{1}$," J. Math. Soc. Japan, v. 25, 1973, pp. $565-590$.

7. B. M. Budak, E. N. Solov'eva \& A. B. UspenskiI, “A difference method with smoothing of coefficients for the solution of the Stefan problem," Ž. Vyčisl. Mat. i Mat. Fiz., v. 5, 1965, pp. 828-840. (Russian)

8. L. Caffarelli \& L. C. Evans, "Continuity of the temperature in the two-phase Stefan problem," Arch. Rational Mech. Anal. (To appear.)

9. P. G. Ciarlet, The Finite Element Method for Elliptic Problems, North-Holland/American Elsevier, Amsterdam/New York, 1978.

10. J. F. Ciavaldini, "Analyse numérique d'un problème de Stefan à deux phases par une méthode l'éléments finis," SIAM J. Numer. Anal., v. 12, 1975, pp. 464-487.

11. A. Damlamian, "Some results on the multi-phase Stefan problem," Comm. Partial Differential Equations, v. 2, 1977, pp. 1017-1044.

12. E. DI BenedetTo, Continuity of Weak Solutions to Certain Singular Parabolic Equations, MRC Tech. Report 2124, Madison, Wisc., 1980.

13. J. Douglas, JR., T. Dupont \& L. Wahlbin, “Optimal $L^{\infty}$ error estimates for Galerkin approximations to solutions of two-point boundary value problems," Math. Comp., v. 29, 1975, pp. 475-483.

14. N. Dunford \& J. Schwartz, Linear Operators, Vol. I, Wiley, New York, 1957.

15. A. Friedman, “The Stefan problem in several space variables," Trans. Amer. Math. Soc., v. 133, 1968 , pp. 51-87.

16. E. Hille \& R. S. Phillips, Functional Analysis and Semigroups, Amer. Math. Soc. Colloq. Publ., vol. 31, Amer. Math. Soc., Providence, R. I., 1957.

17. J. JEROME, “Nonlinear equations of evolution and a generalized Stefan problem," J. Differential Equations, v. 26, 1977, pp. 240-261.

18. J. JEROME, "Existence and approximation of weak solutions of nonlinear Dirichlet problems with discontinuous coefficients," SIAM J. Math. Anal., v. 9, 1978, pp. 730-742.

19. C. Johnson \& V. Thomé, "Error estimates for some mixed finite element methods for parabolic type problems," RAIRO Anal. Numér., v. 15, 1981, pp. 41-78.

20. S. Kamenomostskaja, “On the Stefan problem,” Mat. Sb., v. 53, 1961, pp. 489-514. (Russian)

21. T. Kato, "Linear evolution equations of hyperbolic type II," J. Math. Soc. Japan, v. 25, 1973, pp. 648-666.

22. S. N. KRUzhKov, "First order quasilinear equations in several independent variables," Math. USSR Sb., v. 10, 1970, pp. 217-243.

23. O. Ladyzhenskaya, V. Solonnikov \& N. Ural'CeVA, Linear and Quasilinear Equations of Parabolic Type, Transl. Math. Monographs, vol. 23, Amer. Math. Soc., Providence, R. I., 1968.

24. A. LAZARIDIs, "A numerical solution of the multidimensional solidification (or melting) problem," Internat. J. Heat Mass Transfer, v. 13, 1970, pp. 1459-1477.

25. J. L. Lions, Quelques Méthodes de Resolution des Problèmes aux Limites non Linéaires, Dunod, Paris, 1969.

26. G. H. MeYer, “Multidimensional Stefan problems," SIAM J. Numer. Anal., v. 10, 1973, pp. $522-538$

27. J. Nitsche, $L^{\infty}$ Convergence of Finite Element Approximations, Proc. Second Conf. on Finite Elements, Rennes, France, 1975.

28. R. Rannacher, “Zur $L^{\infty}$-Konvergenz linearer Finiter Elemente," Math. Z., v. 149, 1976, pp. 69-77.

29. Michael E. Rose, "Numerical methods for flows through porous media. I," Math. Comp. (submitted). Also available as Argonne National Laboratory Report.

30. MiLton E. Rose, "A method for calculating solutions of parabolic equations with a free boundary," Math. Comp., v. 14, 1960, pp. 249-256. 
31. L. Rubenstein, The Stefan Problem, Transl. Math. Monographs, vol. 27, Amer. Math. Soc., Providence, R. I. 1971.

32. A. A. Samarskii \& B. D. Moiseenko, "An efficient scheme for (the) thorough computation in a many dimensional Stefan problem," Ž. Vyčisl. Mat. i Mat. Fiz., v. 5, 1965, pp. 816-827.

33. A. H. Schatz \& L. B. Wahlbin, "On the quasi-optimality in $L_{\infty}$ of the $\dot{H}^{1}$-projection into finite element spaces," Math. Comp., v. 38, 1982, pp. 1-22.

34. R. Scott, "Optimal $L^{\infty}$ estimates for the finite element method on irregular meshes," Math. Comp., v. 30, 1976, pp. 681-697.

35. S. L. Sobolev, Applications of Functional Analysis in Mathematical Physics, Transl. Math. Monographs, vol. 7, Amer. Math. Soc., Providence, R. I. 1963.

36. A. Solomon, "Some remarks on the Stefan problem," Math. Comp., v. 20, 1966, pp. 347-360.

37. G. Strang, "Approximation in the finite element method," Numer. Math., v. 19, 1972, pp. 81-98.

38. G. Strang \& G. Fix, Analysis of the Finite Element Method, Prentice-Hall, Englewood Cliffs, N. J., 1973.

39. J. A. Wheeler, JR., Simulation of Heat Transfer from a Warm Pipeline Buried in Permafrost, Proc. 74th National Meeting AIChE, March 1973.

40. J. A. WheEleR, JR., "Permafrost thermal design for the trans-Alaska pipeline," in Moving Boundary Problems (Wilson, Solomon, Boggs, eds.), Academic Press, New York, 1978, pp. 267-284.

41. J. A. WheEler, JR., Personal communication. 